\title{
Linking Information and People in a Social System for Academic Conferences
}

Peter Brusilovsky, Jung Sun Oh, Claudia López, Denis Parra, Wei Jeng

School of Information Sciences

University of Pittsburgh

135 North Bellefield Avenue,

Pittsburgh, PA 15260

\begin{abstract}
This paper investigates the feasibility of maintaining a social information system to support attendees at an academic conference. The main challenge of this work was to create an infrastructure where users' social activities, such as bookmarking, tagging, and social linking could be used to enhance user navigation and maximize the users' ability to locate two important types of information in conference settings: presentations to attend and attendees to meet. We developed Conference Navigator 3, a social conference support system that integrates a conference schedule planner with a social linking service. We examined its potential and functions in the context of a medium-scale academic conference. In this paper, we present the design of the system's socially-enabled features and report the results of a conference-based study. Our study demonstrates the feasibility of social information systems for conference support. Despite the low number of potential users and the short timeframe in which conferences take place, the usage of the system was high enough to provide sufficient data for social mechanisms. The study shows that most critical social features were highly appreciated and used, and provides direction for further research.
\end{abstract}

Keywords: Social information system, social linking, conference support system

\section{INTRODUCTION}

Over the last few years, social information systems have improved our ability to access information in many areas. Social linking systems, social tagging systems, and collaborative recommenders all leverage the power of a community by collecting various kinds of user-contributed information (such as links, tags, or ratings) and use this information to enrich our ability to find relevant products, links, or movies, as well as enabling users to stay in touch with friends and contacts.

Since many researchers and practitioners are engaged in work on social information systems, it was natural to see the creation of social information systems to support professional work. For example, there are social linking systems, like LinkedIn.com or Academia.edu; social bookmarking systems for research papers, like CiteULike.org or BibSonomy.org; systems to share information about research conferences, like 
WikiCFP.org; and even research paper recommenders (McNee et al., 2006; Middleton et al., 2004; Sugiyama and Kan, 2010). These systems have all increased our ability to find relevant papers and collaborators, and have become an everyday feature of academic life.

This paper explores the feasibility of creating and maintaining a social information system to achieve a related, but somewhat more challenging goal—supporting a research conference.

A conference could be considered as an extreme case of information and social linking. Conference attendees are challenged to find the most important presentations and network with people who are relevant to their research field in just 2-3 days while simultaneously navigating between various presentation tracks and social events spread out over the conference site. With its ability to help in finding relevant people and presentations, a social information system could provide a unique value in this context.

However, building a successful conference system that includes these socially-based features might be a real challenge, due to the limited nature of the community behind the system. Social information systems face a wide range of challenges to maintain active streams of user-generated content, such as attracting new contributors, as well as retaining current ones (Kraut and Resnick, 2012). One of the key factors of this challenge is the well-known tendency for only a small fraction of users to become regular contributors (Cummings et al., 2002). The 1\% rule of active participation has been commonly assumed among practitioners, and was recently confirmed by researchers (van Mierlo, 2014). This rule states that while $90 \%$ of the users of a social information system remain passive observers or readers of content, $9 \%$ of the users contribute content somewhat sparsely, and only $1 \%$ of the user base are active contributors who provide the vast majority of content to the systems. This is still more than sufficient for many social information systems, such as LinkedIn, Twitter, and Wikipedia, which are fueled by large, frequently worldwide communities and collect contributions over a long period of time.

However, if this rule is applied in the context of conference support systems, then their socially-based features are likely to fail. One percent of the total number of conference attendees (which often ranges from 100 to 3,000) is probably too small for a social information system to gather enough collective data to perform well (especially over a very short period) and thrive. Indeed, other kinds of audience-based social information systems, namely systems for neighborhoods, often struggle to become viable, due to the challenge of a small target audience (Carroll and Rosson, 2013).

The work reported in this paper explores social conference support systems from the positions of both value and feasibility. Which components and features of a social conference support system are most important for conference attendees? How we can use community wisdom accumulated in a typical social system (such as bookmarks, tags, or social connections) to provide a better access to information about conference presentations and attendees to the system users? Can attendees of a typical academic conference generate a sufficient volume of contributions to fuel social mechanisms? How can such a system and its social features be used in the context of a large academic conference?

Our Conference Navigator project attempts to answer these questions in a practical way by developing incrementally more complex social conference systems and exploring 
them in real academic conferences. This paper presents our study of Conference Navigator 3 (CN3), the third version of our conference support system, which introduced a range of social features to provide extensive linking and rich information access in the system. It presents the main components and features of $\mathrm{CN} 3$ that are related to linking and information access, analyzes users' contributory and navigation activities, summarizes user feedback about social system features, and discusses what we learned from the process.

\section{RELATED WORK}

The problem of information support for research conferences is certainly much older than social systems, and even computer information systems. For years, providing conference attendees with two principal information resources, the conference program and the list of attendees, has been the primary mechanism to address this problem. The conference program provided a schedule of presentations and other events, and was sometimes extended with additional information like presentation abstracts and biographies of the most important speakers. The list of attendees was provided to support social networking. It allows the participants to see who is in attendance, their affiliation and position, and, at some conferences, how to contact each attendee.

Naturally, the advancement of information technology challenged researchers to go beyond the traditional printed program and list of attendees to offer more help in finding relevant presentations and social networking. In this section, we offer a brief review of these attempts. The space of conference support systems is quite diverse, with a range of different models that use various combinations of hypertext and websites, information kiosks and public displays, and sensor badges and mobile devices. To offer some guidance in understanding this work, we group systems by their key purpose, separately reviewing systems with a primary focus on finding presentations to attend and systems that focus on supporting social networking. To some extent, this division represents the prospects of different research communities, with Hypertext researchers focused mostly on presentation finding, while the HCI and Ubiquitous Computing community focused on social networking opportunities. We also discuss several separate issues that cut across these two groups and are of special relevance to our work.

\subsection{Which presentation I should attend?}

The research in this direction has focused on augmenting the presentation-finding function of a traditional conference program. It is also the oldest stream of research on conference support. The pioneering work in this area was done in a hypertext community by turning a conference program from text to hypertext. The most known example of this work is CHI'89 InfoBooth (Salomon, 1990), a Hypercard-based conference information system that was piloted at CHI'89, well before the advent of the Internet. The emergence of WWW as universal hypertext just years after this pioneering work turned this approach from research into practice.

Since the early days of the Internet, it became more and more typical for a conference to publish its program in advance on its website. The next major research advancement in this direction was introduced 11 years later by Sumi and Mase (2001) who moved a hypertext-like conference information space for JSAI 2000 to a mobile application for 
PalmGuide, which made this information nearly as portable as the printed conference program - but more powerful. This idea has also quickly turned from research into everyday practice. Gradually, many large-scale conferences migrated from simple static web pages that displayed programs to interactive online systems and mobile apps that provide better search and navigation capabilities. Nowadays, many large research and trade conferences offer both Web-based interactive online programs and mobile apps that provide access to conference information on the go. This trend encouraged the appearance of several companies that offer conference-support software (Farnham et al., 2009).

Meanwhile, the focus of the research community moved from simply providing a hyperspace with conference information to helping users find the most relevant presentations. Given its origins in the Hypertext community, this work has explored a combination of social navigation and recommendation approaches. The original Conference Navigator system made the first attempt to offer personalized guidance in a hyperspace of conference presentations (Farzan and Brusilovsky, 2007; Farzan and Brusilovsky, 2008). This work was based on ideas of social navigation (Dieberger et al., 2000), a concept that was introduced in late 1990 as an approach that uses traces of past user activities in a system as a "community wisdom" to guide future users of the system.

Conference Navigator enhanced the usual browsing of a Web-based conference program with the ability to add comments and ratings for each presentation, as well as the ability to add a presentation to a user's personal schedule. It also encouraged users to join one or more topic-oriented sub-communities. A user's browsing, rating, commenting, social linking, and scheduling behavior was monitored and credited to the current user community.

Using this data, the system was able to identity which presentations were most frequently checked and scheduled within each sub-community. This information was used to label the most popular presentations in the schedule and to produce a list of recommended presentations to other users in the same community. The use of the system at the E-Learn 2006 and 2007 conferences demonstrated the value of social navigation within a conference context.

The second version of Conference Navigator, CNII (Wongchokprasitti et al., 2010) (Brusilovsky et al., 2010) enhanced the original set of social navigation techniques with the use of tags (which are among the most useful types of community wisdom) and added the ability to track users' actions, not only at the group level, but also at the individual level. In turn, this data allowed developing and evaluating some more advanced social navigation approaches. Each presentation was now annotated with a much broader social offprint of past users to help future users in judging the presentation as relevant. For example, the information about a presentation included the list of users who bookmarked it, the list of tags, the list of communities that scheduled the presentation, and any user notes about the session. CNII was used at several conferences between 2008 and 2010, in which different kinds of recommendation approaches were evaluated, including tag-based recommendations (Brusilovsky et al., 2010).

The use of social navigation and recommendations in a conference context has gradually become quite popular, with several more recent systems using different combinations of these features. For example, the Event Maps system (Wang et al., 2010) offered a simple 
version of social navigation to help with presentation scheduling (displaying the number of users who bookmarked a talk). TalkRadar, a component of Conferator (Atzmüller et al., 2011) used the author's tags for talk finding. Several projects reviewed in a separate subsection explored various presentation recommendation approaches in a conference context (Kim et al., 2013; Pham et al., 2012; Yaw Asabere et al., 2014). Finally, the Cobi project (Kim et al., 2013) attempted to enhance the scope of social navigation. While it follows other similar projects in collecting community data and applying it to recommending presentations, it also pioneered the use of such "crowdsourcing" to help conference organizers create a better conference schedule.

In the context of this work, Conference Navigator $3(\mathrm{CN} 3)$ represents a further step in the stream of research on social navigation and assisting users in finding relevant presentations. By integrating presentation scheduling and social linking components, CN3 has created a much wider hyperspace to offer broader social navigation support for users. Most notably, user-created social links supported by $\mathrm{CN} 3$ have introduced yet another kind of "community wisdom" that has been leveraged by social navigation, visualization, and recommendation approaches. By providing more navigation opportunities and better guidance, $\mathrm{CN} 3$ also attempted to become more appealing and increase both the fraction of engaged users and the volume of their activity, which is a vital issue for all social navigation systems fueled by "community wisdom" (Bhardwaj et al., 2014).

\subsection{Who should I talk to?}

Research on supporting social linking opportunities has taken a considerably different approach. While several projects have made a direct attempt to enhance the traditional list of attendees using Web and hypertext (Atzmüller et al., 2011; Churchill et al., 2004) (Farnham et al., 2009), the majority of work has focused on enhancing user opportunities to meet in real space (rather than in hyperspace) by exploring various combinations of sensor badges and public displays. Badges are typically used to track user contacts, location on the conference floor, and a user's presence next to a public display. In turn, displays are typically used as "community mirrors" to show either a static or a dynamic picture of the user community or as "interactors" to encourage and mediate user communication. Past work in this direction can be roughly grouped into three main streams (reviewed below) with some work that crosses the borders.

The first stream could be classified as a social networking stream, which most closely resembles social networking sites (Ellison, 2007). These systems usually offer users an opportunity to create profiles that provide relevant information about themselves. They also provide an online space where these profiles can be explored (Atzmüller et al., 2011; Chin et al., 2012; Churchill et al., 2004; Farnham et al., 2009; Sumi and Mase, 2001). The presence of an online space also makes it natural for the systems in this group to provide some simple version of an online conference program and a basic discussion board (Churchill et al., 2004; Farnham et al., 2009; Sumi and Mase, 2001). Some of these systems also offer basic people recommendations (Chin et al., 2012; Sumi and Mase, 2001).

A considerable subset of projects belonging to the social networking stream actively explore the use of public displays and sensors. For example, CHIplace and CSCWplace (Churchill et al., 2004) have used public displays to encourage public exploration of the 
social online space. Find \& Connect (Chin et al., 2012) uses location-tracking sensor badges to provide location-based exploration of contacts (who is next to me, who is further away). Since badges are also able to track user session attendance, the system also can show which presentations have been attended by both the target user and a prospective contact.

The second stream could be classified as a ubiquitous interaction stream. It is based on the idea that a combination of public displays and badges could be used to sense nearby users (Dey et al., 1999) and provide information about them to enhance social interaction. This information could simply be a conversation starter, or even some more advanced information that focuses on prospective collaboration. In some cases, it could be information about a single user: for example, AutoSpeakerID (McCarthy et al., 2004) displays information about attendees who to ask questions after a presentation. Ticket2Talk, which was developed by the same team, presents interest profiles of attendees in a coffee break area. More popular in this stream, however, is the use of public displays as "interactors" between two or more people.

As mentioned above, this direction was pioneered by (Sumi and Mase, 2001) with the AgentSalon system that allowed up to four users to share their conference experience. Neighbourhood Window (McDonald et al., 2008) piloted an attractive idea of similarity visualization, as it shows how up to four nearby users are connected through their topics of interest. DeaiExplorer (Konomi et al., 2006) expanded the idea of similarity visualization by adding shared co-authors or cited authors to the similarity visualization.

Finally, the third stream that could be classified as conference dynamics, uses the ability to trace user communication or location to present a dynamic picture of the conference as a whole on a "community mirror" public display. This stream was started by Meme Tags (Borovoy et al., 1998), which displayed the dynamics of "meme" spreading. IntelliBadge ${ }^{\mathrm{TM}}$ focused on tracking user location and used public displays to show conference statistics and the dynamic distribution of attendees over conference rooms. Sociopatterns (Cattuto et al., 2010) returned back to tracing user contacts, but used a much more advanced approach to visualize user communications. Later, Conferator (Atzmüller et al., 2011) used the infrastructure developed in Sociopatterns to track and display both contacts and the location of volunteered delegates. It is interesting to note that though these systems began as a way to encourage social networking, most recent projects also use data collected by sensors to study conference dynamics. Some interesting findings in this area can be found in (Macek et al., 2012).

Within this context, the $\mathrm{CN} 3$ system belongs to the first stream. It provides a solution for Web-based social networking with user profiles and presentation discussions. Its contribution to this stream is the ability to establish direct user connections, and the application of connection data to offer both user and presentation recommendations. CN3 also pioneered comparative user information presentations, based on the ideas of social navigation: a target user could explore the planned schedules of other users in comparison with his or her own schedule in both text and visual form. These features can serve as a tool to find both likeminded users and relevant presentations.

\subsection{Integrated systems}

Since the integration of presentation finding and social networking support is one of the distinct features of $\mathrm{CN} 3$, it is important to mention other projects that have attempted to 
offer both kinds of support. JSAI 2001 Digital Assistant (Sumi and Mase, 2002) is a clear pioneer in this area. However, it provided only basic support for presentation finding, and it was a collection of several loosely connected tools, rather than an integrated system. Another notable system was Conferator (Atzmüller et al., 2011), which used two independent subsystems: TalkRadar, for presentation finding, and PeerRadar, for social networking. While the Conferator as a whole was balanced, the loose connection between its components did not allow the system to fully leverage both kinds of information. In contrast, CNII (Brusilovsky et al., 2010) and Find \& Connect (Chin et al., 2012) were truly integrated systems, but were not really balanced. CNII had elaborate support for presentation finding and basic support for networking, while Find \& Connect had basic presentation finding and elaborate social networking.

In this context, the $\mathrm{CN} 3$ system presented in this paper is the first attempt to create a balanced integrated conference support system that provides advanced support for both presentation finding and social networking. Moreover, it extensively used information from one of its "sides" to enhance the other one: namely, social links created in the "people space" were used to provide social navigation and recommendations in the "presentation space," while bookmarks and tags created during presentation exploration enhance social navigation and recommendations in the "people space."

\subsection{Information visualization for conferences}

The use of information visualization for conferences was originally motivated by public displays that provided ample space, while simultaneously demanding information presentation in a form that is easy for all conference attendees to understand. More recently, the increased quality of laptop displays has made this approach increasingly more attractive for users. Over the past 15 years, conference support systems explored three primary types of visualization: location-based, network-based, and time-based.

The simplest location-based visualization was typically used to show session location and/or user distribution on public displays (Atzmüller et al., 2011; Cox et al., 2003). The more advanced network-based visualization was pioneered by JSAI 2001 Digital Assistant (Sumi and Mase, 2002), which offered a view of conference information as a network of people and presentations. Neighbourhood Window (McDonald et al., 2008) and DeaiExplorer (Konomi et al., 2006) also provided social network-style visualizations, but the visualizations focused on only two to four communicating users. Time-based visualization in tabular or timeline form was used by several projects to show the overall conference program "at a glance" (Wang et al., 2010) and to display papers selected by the user in a broader context (Atzmüller et al., 2011; Wang et al., 2010).

More recently, some more advanced visualization approaches have been used to visualize the space of conference presentations. Conference Explorer (Windhager et al., 2014) and Altmetrics (Kraker et al., 2014) suggested different versions of topic-based visualization, while TalkExplorer (Verbert et al., 2013) and SetFusion (Parra and Brusilovsky, 2015) explored two versions of set-based visualizations. In this context, the visualization used by $\mathrm{CN} 3$ could be classified as time-based. The contribution of this project lies in enhancing program visualization with social navigation and social comparison by allowing users to visually compare their schedules with the schedules of other users. 


\subsection{Recommender systems for conferences}

Recommender systems used in a conference context are one of the newest approaches to support conference attendees. The first attempts to recommend presentations (Farzan and Brusilovsky, 2008; Sumi and Mase, 2001) and people to meet (Farnham et al., 2009; Farrell et al., 2005) were very simple by the standards of the recommender-systems field. Up to our knowledge, CNII was the first project that introduced and evaluated advanced presentation recommender approaches in the context of a conference support system (Brusilovsky et al., 2010). The CN3 system presented in this paper provided further advancement by integrating both people and presentation recommendations, and by using social links for both people and presentation recommendation (Lee and Brusilovsky, 2012).

More recent projects focused solely on recommendation algorithms rather than on building conference support systems (Pham et al., 2012; Scholz et al., 2014; Yaw Asabere et al., 2014) with some of these projects exploring implicit social connections, as could be tracked by sensors. In this context the $\mathrm{CN} 3$ project still stands out as one the first implementations of advanced recommender approaches in a conference support system and as the only extensive exploration of explicit social links for presentation recommendations (Lee and Brusilovsky, 2014).

\section{Pilot study}

As mentioned above, $\mathrm{CN} 3$ is the third version in the Conference Navigator line. It benefits from the experience of $\mathrm{CN}$ and CNII, and represents the result of a careful redesign of its previous versions that has focused on expanding the social features of the system and enhancing social navigation capabilities. The main goal of this expansion is to leverage the association between presentations and users. To do that, the system uses the user activities, such as scheduling and tagging, as well as the social connections among users, to create new mechanisms to support users in finding both interesting presentations and people with shared interests.

Since most of these features were new at the time of launching CN3 and their value to the end users was not clear, we started the redesign process with a field pilot study that was performed with a modified version of $\mathrm{CN} 2$. This version included some crude implementation of $\mathrm{CN} 3$ ideas (such as adding attendees as first-class objects to enable user-based navigation), as well as existing $\mathrm{CN} 2$ features. This pilot study had two primary objectives: first, to assess the value of existing features and identify features to retain in $\mathrm{CN} 3$; and second, to solicit user input on additional social features and functionalities to be included or expanded in CN3.

The pilot study was performed at the 2010 Annual Meeting of the Association for Information Science and Technology (hereafter ASIS\&T 2010). During ASIS\&T 2010, conference attendees were invited to use our system (Figure 1). Out of 550 attendees, 58 users used the system sufficiently deeply to bookmark at least one presentation. At the end of the conference, all attendees who used the system were invited to fill out a paper survey. In total, we collected 19 surveys. 


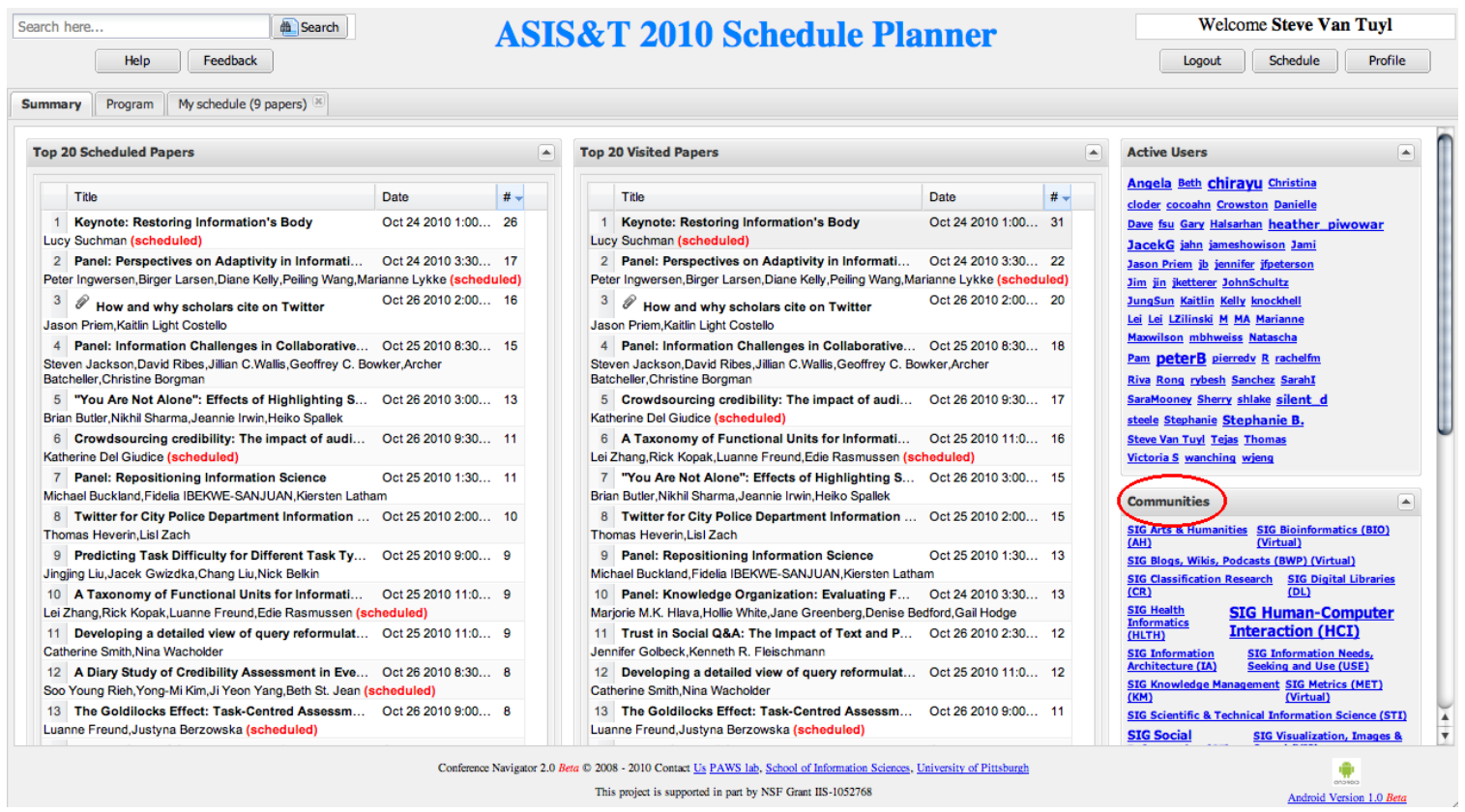

Figure 1. The pilot system employed in ASIS\&T for the pre-study.

The survey included two broad categories of questions: one for evaluating the existing features of the pilot system, and the other for assessing the potential usefulness of planned expansions that had not been incorporated in the pilot system. In both cases, the participants were asked to what extent a specific feature either is or would be useful, using a 5-point agree/disagree Likert scale.

As Table 1 shows, the majority of participants found the key social features in the pilot system (such as seeing other users who bookmarked a presentation, seeing other users' schedules, seeing most popular presentations) to be useful, along with the basic conference support functionalities (such as a personal schedule page). Surprisingly, there were mixed responses regarding the usefulness of presentation recommendation, with many users neither agreeing nor disagreeing that the feature is useful. As for potential areas of expansion (items marked by an asterisk (*) in Table 1), the participants liked the idea of getting visual cues to mark those events (presentations) that he/she bookmarked (added to his/her schedule) in the global view of the conference program. A calendar view of the conference program/schedule also got mostly positive responses. Many participants agreed that connecting with other users with shared interests within the conference system would be useful.

Overall, the pilot study confirmed both the feasibility and desirability of moving forward with our ideas for $\mathrm{CN} 3$, and allowed us to prioritize the implementation of features, based on user feedback. 
Table 1. User Feedback: Usefulness of existing/prospective features

\begin{tabular}{|l|c|c|c|}
\hline Usefulness of & Agree (\%) & Neither (\%) & Disagree (\%) \\
\hline $\begin{array}{l}\text { Visual cues for my scheduled events in } \\
\text { program, event schedule, and other lists* }\end{array}$ & 94 & 0 & 6 \\
\hline Seeing other users' activities & 93 & 7 & 0 \\
\hline Calendar view of conference program* & 82 & 18 & 0 \\
\hline $\begin{array}{l}\text { Having personal schedule page showing } \\
\text { bookmarked presentations }\end{array}$ & 82 & 6 & 12 \\
\hline Assigning tags & 80 & 13 & 7 \\
\hline $\begin{array}{l}\text { Networking with other users with shared } \\
\text { interests* }\end{array}$ & 79 & 21 & 0 \\
\hline Seeing other users' schedules & 74 & 27 & 10 \\
\hline Seeing top 20 popular presentations & 64 & 29 & 7 \\
\hline $\begin{array}{l}\text { Seeing users who bookmarked a } \\
\text { presentation }\end{array}$ & 44 & 50 & 6 \\
\hline Recommendation (content-based) & 44 & 38 & 18 \\
\hline Recommendation (tag-based) & & & 0 \\
\hline
\end{tabular}

* Features not yet implemented in the pilot system

\section{Conference Navigator 3 (CN3)}

As mentioned in the introduction, $\mathrm{CN} 3$ attempts to bridge the social and informational aspects of academic conferences. Using several social systems technologies, it integrates and enhances a traditional conference program and list of attendees. The ultimate goal is to use collective intelligence to better help conference attendees to find interesting presentations, as well as find potential new social contacts from among the conference attendees.

From a user's perspective, CN3 works as the integration of a conference calendar and schedule planner with a social linking service. It allows users to browse the conference program, explore information about presentations and attendees, build a personal schedule, and connect to other attendees. In all these contexts, the system supports the users with personalized social guidance, directing their attention to most interesting people and presentations. To stress the innovative social features of $\mathrm{CN} 3$, the system description presented below is split into three subsections: Social Bookmarking, Social Networking, and Social Navigation and Recommendation.

\subsection{Bookmarking: Discovering Presentations and Building a Personal Schedule}

One of the key goals of $\mathrm{CN} 3$ was to enable users to build a personal schedule for the given conference, while using the personal scheduling information of the whole user community as the key source for expanding system linking and user navigation opportunities. The first step in this process is to provide various ways to browse and explore presentations and to encourage and facilitate the creation of personal schedules. $\mathrm{CN} 3$ provides a range of tools to locate relevant presentations, starting with various ways 
to examine the program that shows the scheduled events and presentations, as well as ways to add events and/or presentations of interest to their personal schedules.

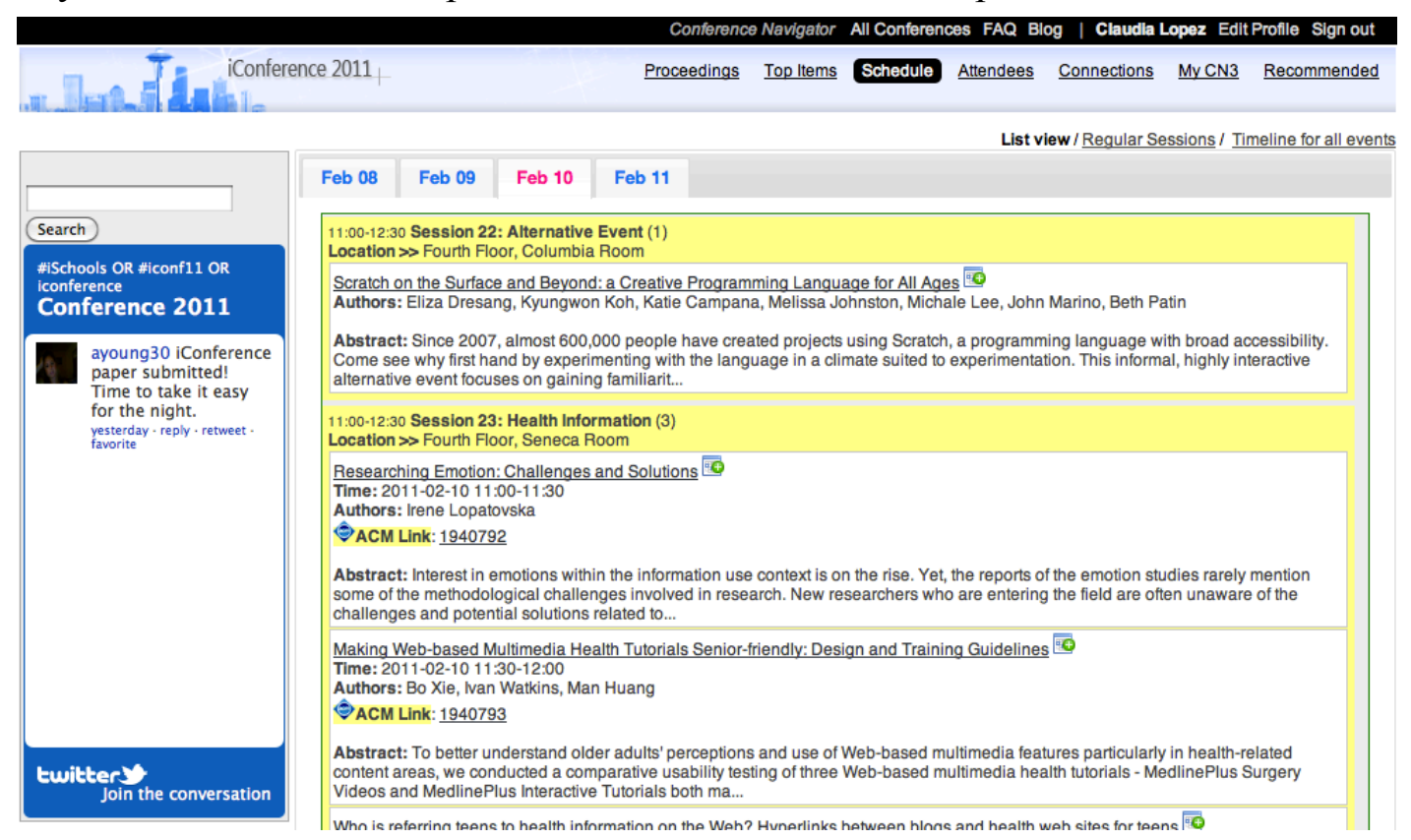

Figure 2. A traditional view of a conference program

The ultimate way to discover relevant presentations is provided by views of the conference program. CN3 supports a traditional day-by-day program view (as Figure 2 shows) where each day shows the list of sessions and presentations ordered by time. In addition, CN3 provides a novel time-based program visualization that supports several advanced methods of presentation search and exploration (Figure 3). The calendar view presents the entire conference program subdivided by days. The structure of each day is shown as a table of parallel sessions, where individual presentations are shown as timebars that represent the duration of each presentation and its position in the session. The color of each timebar indicates whether the target user scheduled the corresponding presentation. More information about each presentation could be obtained by mousing over the timebar or by expanding a session cell (Figure 3 ).

The holistic nature of program visualization turns this view into an important tool for discovering and scheduling relevant presentations. The visualization supports this functionality through filtering. Filtering allows for highlighting sessions and presentations of interest while fading out other sessions, and thus supports examining presentations of interest in the global conference context. The latter is important, since scheduling decisions depend on both a presentation's relevance and its position in the program.

The view provides five filters. The personal filter allows a user to highlight his/her personal schedule. It is analogous to circling or otherwise marking a session on a paperbased program, a strategy commonly used by conference attendees to remind them of where they intend to go. The search filter highlights presentations that are relevant to a query. The author filter enables users to look for presentations given by specific 
researchers. For example, Figure 3 shows the program, which is filtered to show Peter Brusilovsky's presentations. The filter has found one of his presentations, and the calendar is shown in the day that the presentation will be presented. The social connection filter and the followed users filter works in a similar way, allowing users to view and compare schedules of a user's social connections within CN3. The work of this filter is explained in section 4.3.

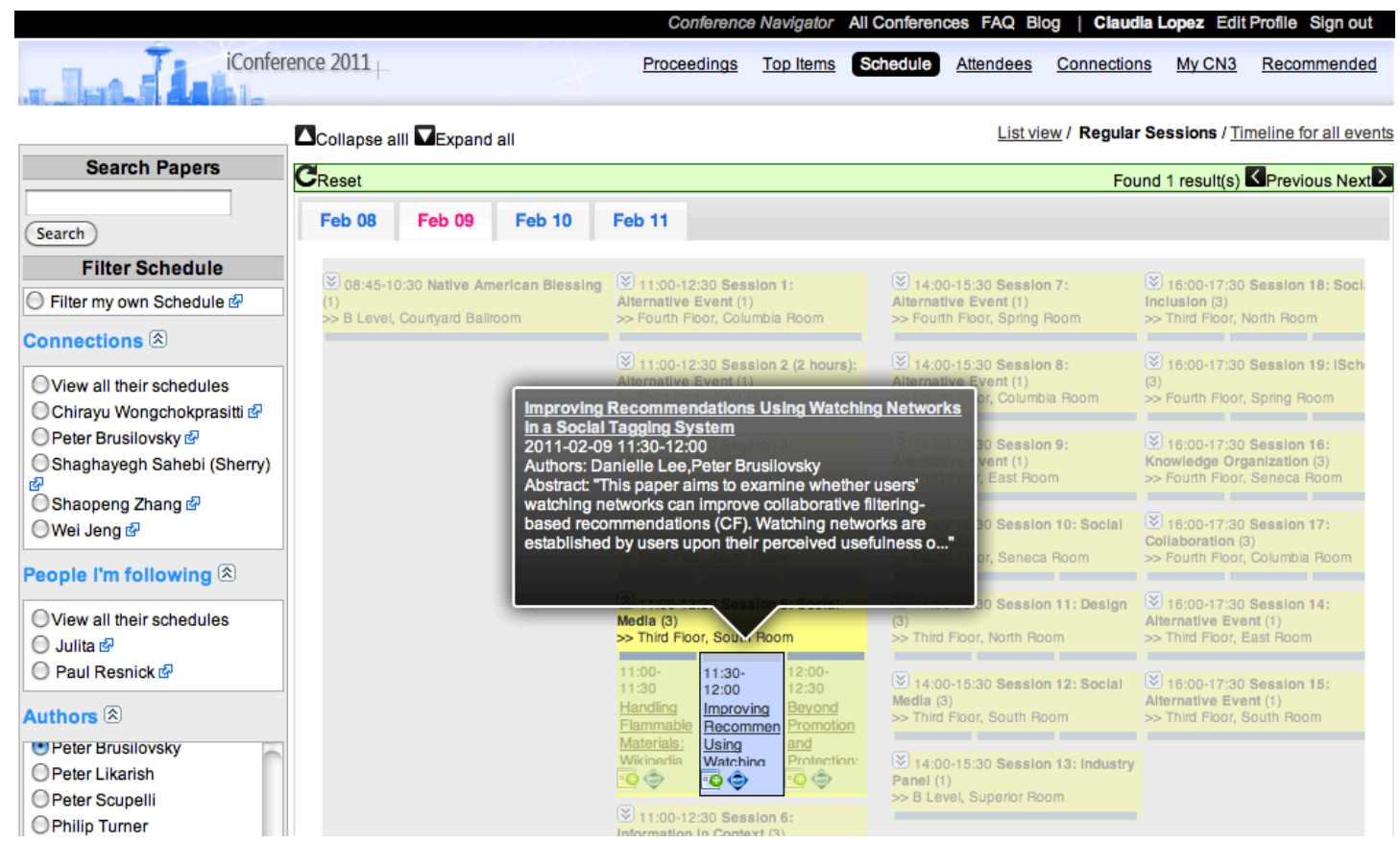

Figure 2. A calendar-based visualization of a conference program. The information about individual presentations could be accessed by expanding a session cell or mousing over each presentation's timebar.

Both program views allows the users to immediately bookmark a presentation of interest, or click a link to the presentation to access a page with further details for more information (Figure 4). In addition to the program views explained above, the user can encounter links to presentations in several social views that will be reviewed later. A presentation of interest could be accessed and added to a user's schedule from any of these views. In this version of $\mathrm{CN} 3$, we used the term 'scheduling' and 'bookmarking' interchangeably, although the two concepts are generally different. When a user bookmarks a presentation in $\mathrm{CN} 3$, the presentation is added to his/her personal schedule that can always be accessed through the user profile page. In addition, once a presentation is bookmarked, the history-based navigation support mechanism will mark it as 'scheduled' in any context where links to presentations are shown, including the conference program, proceedings, other attendees' schedules, and other information. Therefore, users can easily see which presentations are scheduled without having to go directly to their schedule page. 


\subsection{Social Networking: Finding and Connecting to Colleagues}

The second key goal of $\mathrm{CN} 3$ is to help users to locate and contact interesting colleagues. To support this goal, the system offers several ways to find relevant colleagues, a dedicated user profile page that allows users to learn more about a potentially interesting attendee and two ways to establish direct connections. As in the case of bookmarks, the connection data contributed by the community is used to offer extended linking and navigation opportunities.

The list of attendees, a traditional conference tool supported by $\mathrm{CN} 3$, provides an ultimate way to locate attendees. This list is only accessible to the attendees of the conference. It shows the name, affiliation, position, and home page of each attendee who has opted to share this information with the rest of attendees. It also displays the number of presentations he/she has scheduled. Each user name in the list of attendees is a link that leads to a user profile page (Figure 5), which provides further information about this user and his/her activities. In addition, CN3 extensively uses social linking (creating new links based on user activities) and social navigation, which causes user links to appear in several other contexts that will be examined below. In each of these contexts, user links lead to the profile pages of the corresponding users.

Once a user lands on the profile page of another user, the target user can initiate two kinds of explicit social links with this user: a bi-directional connection and unidirectional following (as shown at the top of Figure 5). Connection is a mutual relationship that requires a confirmation from the other party, while following is a unidirectional relationship that allows users to simply mark people of interest to follow. The first type is typical for social networking systems, while the second is used primarily in social bookmarking systems.

Once established, both kinds of social links allow users to track other user's activities (if allowed by privacy settings), which gives users the ability to quickly view which conference events they are attending. An easy way to access schedules and other information about socially linked users is the personal profile page (Figure 5) that provides links to both connected and followed users. Each of this link leads to the profile page of the selected user that provides access to that user's schedule and social links (if the privacy settings allow it). This information can be used to discover new relevant presentations and new users to connect with. Another place to access schedules of socially linked users is the program visualization, which offers the ability to visually compare one's own personal schedule with the schedules of both connected and followed users. Both features are reviewed in the next subsection.

While connections and following relations seem to support the same capability of simplifying the process of tracking other users' activities, several CN3 mechanisms (including privacy and recommendations) treat these two types of relations differently. We hypothesized that users might make a 'connection' in CN3 because they do know each other (that is to say, have a real social relationship of some sort), but not necessarily because they share specific research interests. In cases of 'following,' however, it was assumed that, when a user follows others to watch their activities, the motivation is more likely to be informational than social. In that sense, the act of following reflects the user's perception of shared research interests. Due to the different nature of these relationships, these two kinds of links are clearly distinguished in the interface (see Figure 5 and Figure 
6), and the recommendation algorithms of $\mathrm{CN} 3$ use the information about connections and following in different ways.

\subsection{Social Navigation}

One of the main assets of the CN3 system is the information about users, including their bookmarked presentations and connections. In the area of social computing, bookmarks, tags, and social connections are considered as important community wisdom and are used to provide several kinds of social information access (Brusilovsky, 2008). As mentioned above, one of the goals of the $\mathrm{CN} 3$ is an extensive use of socially contributed information to provide additional information links and offer new navigation opportunities. A simple example of social linking shown below is turning bookmarks into two-way social navigation links between information about users and presentations (namely, connecting the world of information and the world of people). However, CN3 attempts to go beyond this simple approach and offers several tools that process bookmarking and linking information to offer the users some more advanced approaches to find relevant information. This section provides an overview of CN3's social navigation and recommendation functionalities.

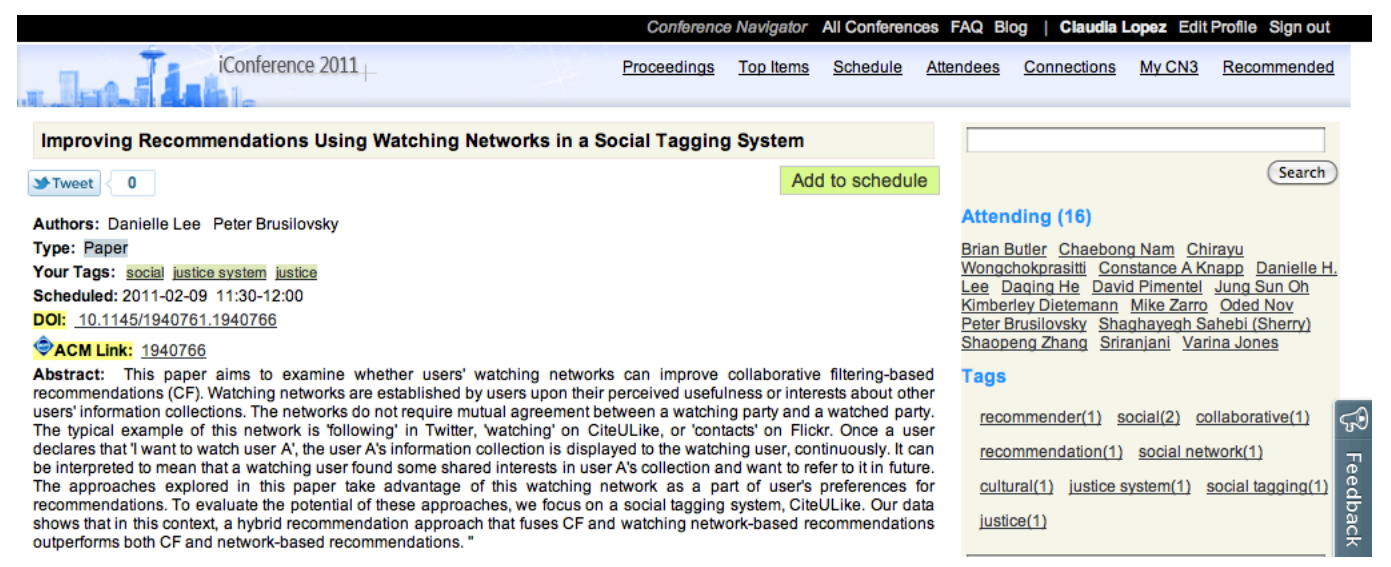

Figure 4. A presentation details page with the list of attending users and tags.

The presentation details page. To complement the list of attendees, CN3 supports several in-context ways to find interesting colleagues. One of these ways is provided by the presentation details page (shown in Figure 4), which is a landing page for a presentation link, in a context where the presentation title is shown. As in several other conference support systems, the main goal of this page is to provide additional details about the presentation that may help users to decide whether the presentation is of interest to them. The traditional metadata information, including the abstract, is shown in CN3 on the left side of this page. The right side shows the social information, including the list of other users who have scheduled the presentation and the list of tags that the users have assigned to the presentation. This information has two functions. On one hand, it helps users to determine whether the presentation is interesting. The fact that this presentation is scheduled by some known attendees or tagged by a relevant tag is important information 
to make this decision. If the presentation is of interest to the user, the user can press the Add to schedule button.

Once a presentation is scheduled, the button changes to "unscheduled," which allows users to remove a presentation from their personal schedule. The page also allows users to add comments and tags to the presentation. Note that the list of users who scheduled the presentation is an excellent tool to locate like-minded users. An attendee who is not yet known to the user, but who scheduled one or more presentations that the user find interesting, is a potential contact or collaborator. In order to help users to identify and learn more about other users who may share interests with them, each name in the list of 'attending' users (users who have scheduled the given presentation) is a clickable link to the user's profile page, where the user's bookmarked presentations and network connections are shown, as well as the basic personal information that the user has provided.

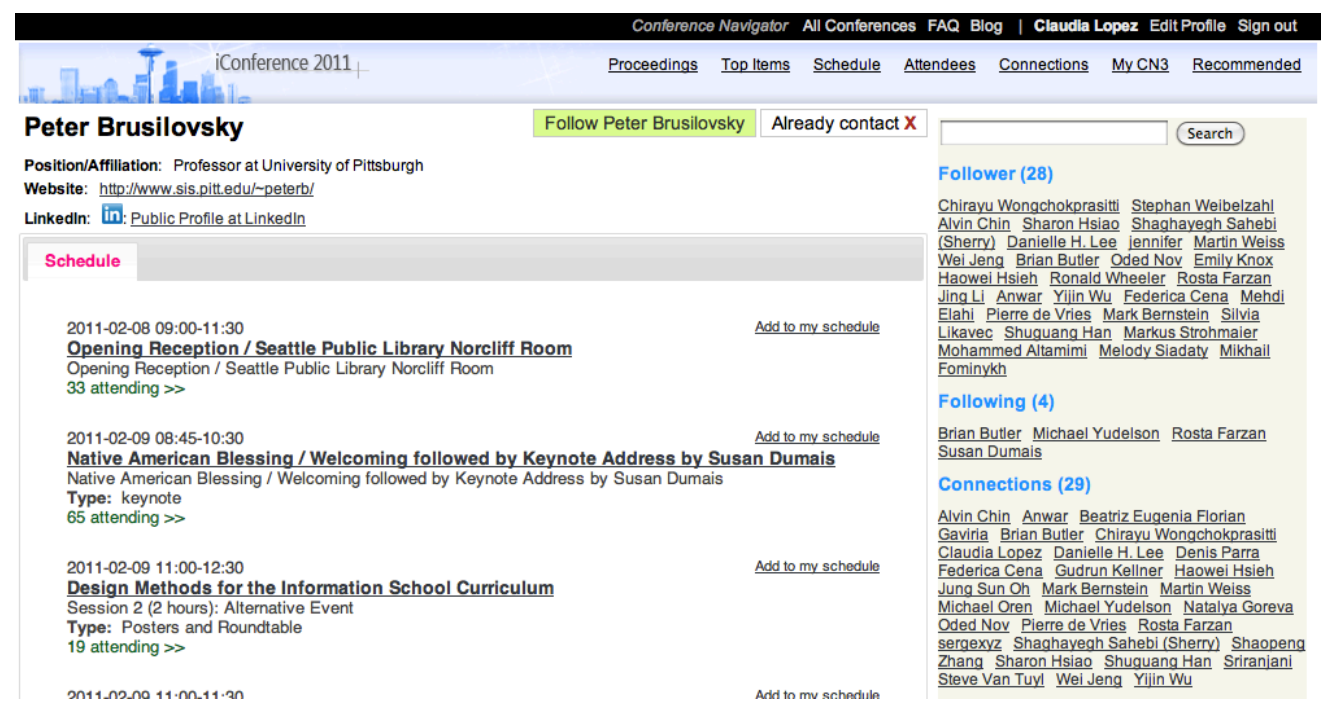

Figure 5. A user's profile page.

The user profile page. For each attendee, the system generates a user profile page (Figure 5 ). This page presents the personal information provided by the user (affiliation, position, and Web page, among other information) and also shows information about the social activities of the user, such as the list of presentations scheduled by the user, the list of social connections, and user tags. The names of social connections who are attending the current conference are highlighted.

The profile page serves two purposes. First, for the user himself or herself, the page can be accessed through the $M y C N 3$ link and serves as a concentrated personal view of the user's conference activities (scheduling and linking). Second, it can be accessed by other users through a link from any context in $\mathrm{CN} 3$ where the user's name is shown and serves as an open profile for that user.. For instance, the list of attendees, various name filters in the calendar view, or the list of other 'attending' users within the presentation view all include links to each named user's profile. The visibility of specific components of an open profile is defined by the owner through the profile's privacy settings. When used by 
an external user, a profile page offers additional social guidance in the form of comparative scheduling. For each presentation in the schedule of the observed user, the system indicates whether this presentation has been already added or not yet added in the observing user's personal schedule. Presentations that are interesting but are not yet added could be added to the user's personal schedule directly from this page. As our data demonstrated, this option emerged as a popular mechanism for adding interesting presentations to individual schedules.

Schedule-Based Comparative Social Navigation. In addition to the local comparative scheduling that each user profile page offers, $\mathrm{CN} 3$ supports broader comparative social navigation and comparative scheduling through its program visualization system, as introduced earlier. This visualization leverages social wisdom collected by the system by allowing a target user to compare her schedule with the schedule of any contact or followed user in the conference program context (Figure 7). The comparison uses two different visual cues to highlight two schedules. The presentations that have been scheduled by the examined user are shown in a bright color (vs. a pale color for the rest of the presentations). Among those presentations, the presentations that have also been scheduled by the target user are indicated by a green bar (vs. a blue bar for the rest of the presentations). This allows the user to easily see which presentations scheduled by the examined user are not yet scheduled (bright and blue), which presentations are already added to both schedules (bright and green), and which presentations are added by the target user, but have not been examined by the user (pale and green).

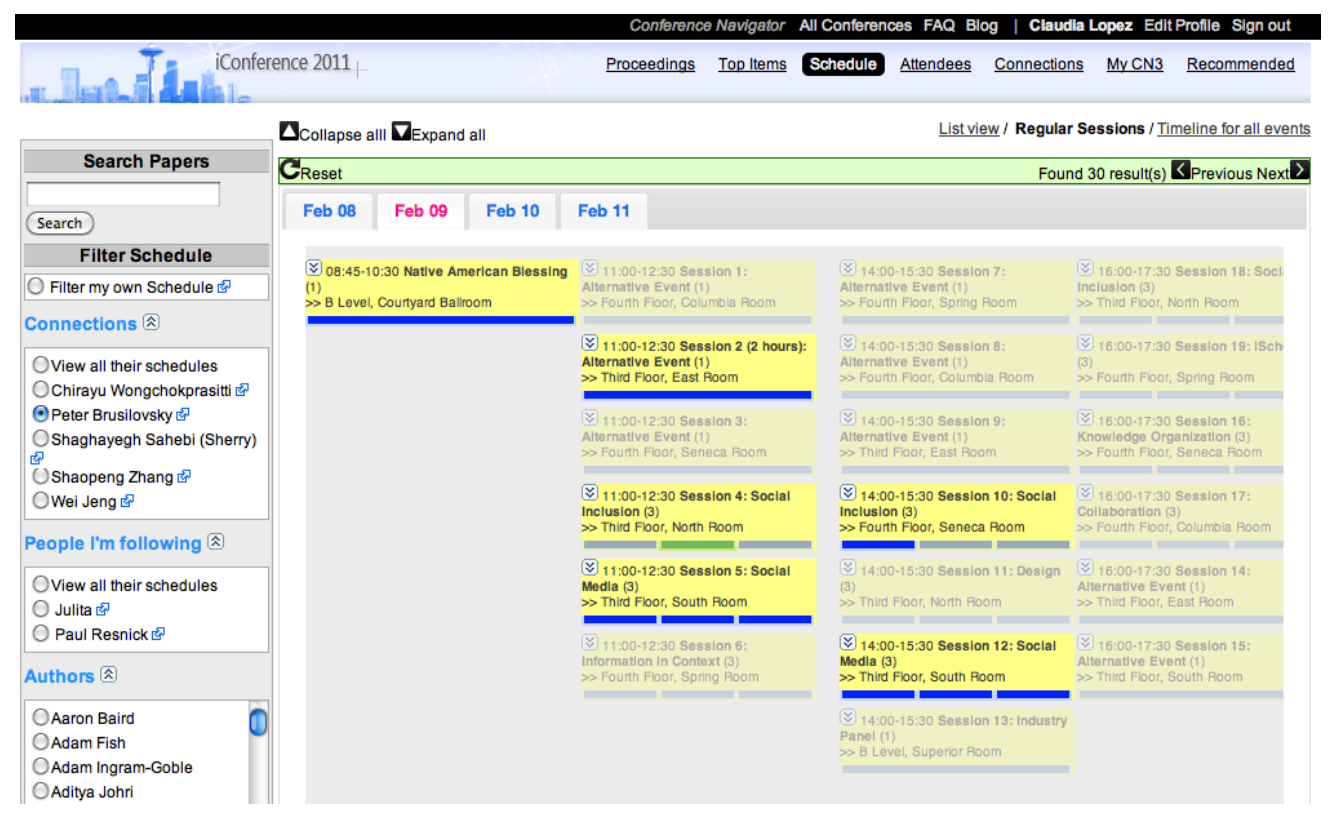

Figure 6. Comparison of a target user schedule with the schedule of the connected user (Peter Brusilovsky)

The Top Items page. Another useful social navigation feature in $\mathrm{CN} 3$ is the Top Items page (Figure 7) that provides another way to display collected social wisdom. The role of 
this page is to track popular events and active people at the conference, making sure that the target user does not miss these events and users. It consists of five presentation areas:

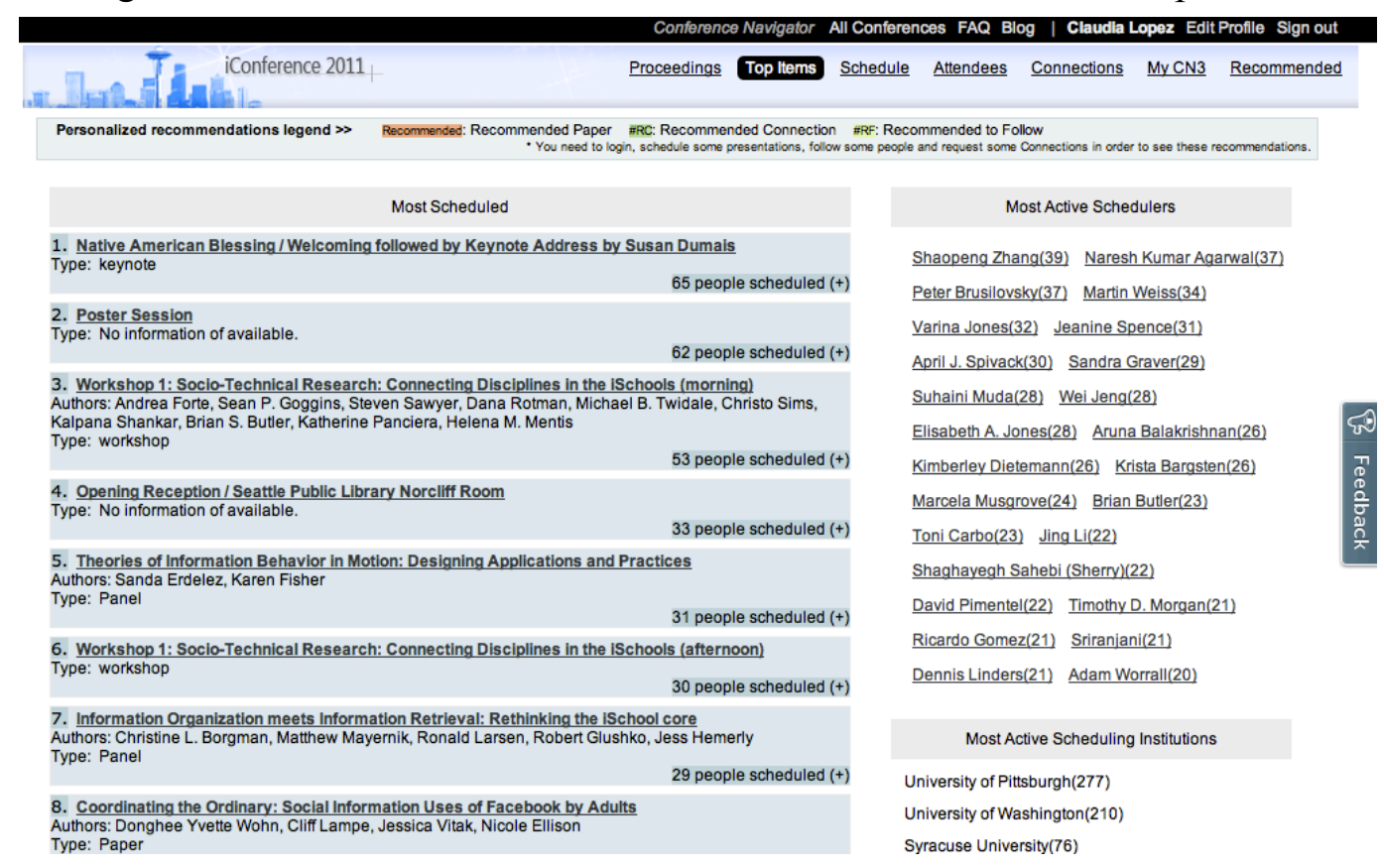

Figure 7. The top items page.

- "Top 25 Scheduled Presentations" counts the number of scheduling activities that all users made and shows the top 25 presentations in a list.

- "Top 25 Scheduling Users" shows the users who have bookmarked more presentations in the conference.

- "Top 25 Scheduling Institutions" counts the number of contributions of all users affiliated to each organization, and shows the 25 institutions whose members have scheduled the most presentations.

- "Most Followed Users" shows the names of the users who are most frequently being followed by other users in the CN3.

- "Most Used Tags" lists the tags that have been more frequently assigned to the conference presentations.

Profile and privacy settings. To balance potential privacy threats that may occur with the extensive use of social navigation, $\mathrm{CN} 3$ offers privacy control and visibility restrictions in the MyCN3 section of the system. This is another place where user connections play an important role. By default, users share their profile information (such as scheduling activities and social links) and their comments with all conference attendees. However, the profile visibility and comments visibility settings can be changed at any time.

The CN3 system supports 5 levels of privacy: user-contributed and other personal information can be set to be visible to (1) everyone; (2) only the user himself/herself; (3) only his/her connections; (4) only registered attendees; and (5) only his/her connections and registered attendees. One of the assumptions here is that the mutual-connection relationship signifies a higher level of mutual trust than a unidirectional "following." If a user chooses to open his/her activities only to those who are connected to him/her (in a 
mutual-connection relationship), then those outside the connection circle would not be able to see his/her activities, even if they 'follow' the user.

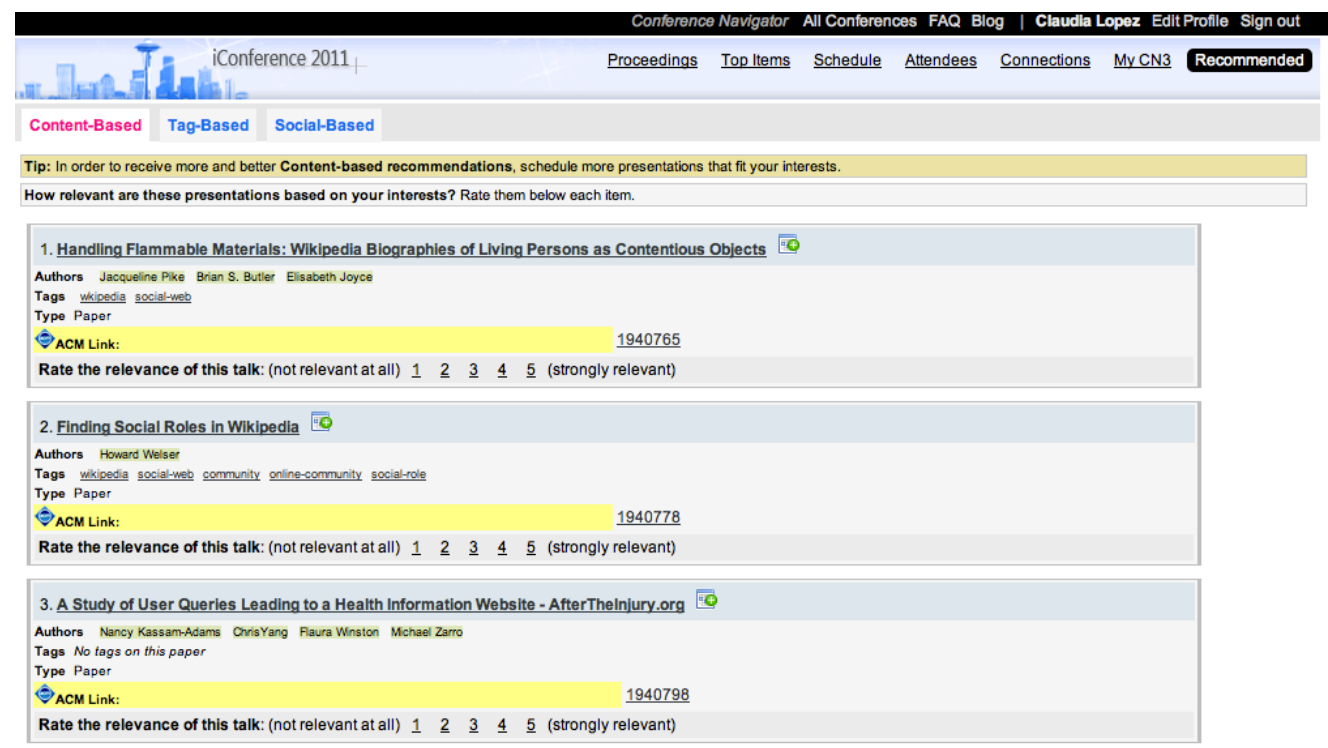

Figure 8. Recommendations in the CN3 system.

\subsection{Recommendations}

In addition to extensive in-context social navigation support, the $\mathrm{CN} 3$ system is also able to generate several kinds of presentation and people recommendations. Although the users in the pilot study did not enthusiastically assess recommendations, we consider them to be a very important component of a conference support system, a pro-active approach to attract users' attention to the potentially most useful presentations and people. All recommendations provided in the system are based on the user data collected by the system; however, the recommendation approaches used in CN3 differ by the kind of activity data used to generate recommendations.

Content-based recommendations are based on the scheduling activities of the target user and consider the title and abstracts of scheduled presentations. As with other contentbased recommendation approaches (Pazzani and Billsus, 2007), it looks for presentations that are similar to those that have been already scheduled by the user and ranks them by this similarity. Tag-based recommendations are somewhat similar to content-based recommendations; however, this approach considers tags used by the target user as a profile of interest and uses tags added by the other CN3 users, rather than abstracts and titles, to characterize conference presentations. Social-based recommendations use coauthorship networks and the social links established within $\mathrm{CN} 3$ to recommend potentially interesting presentations to the users, based on presentations scheduled by their followed users and social connections.

Figure 8 shows the recommendations interface. It includes the option to schedule a recommended presentation and to rate the recommendation on a scale of relevance. User recommendations, suggesting potential social links, are also available. This type of recommendation is also based on a network of co-authorship and social links in CN3 and 
is provided on the user's profile page. The specific algorithms used for each kind of recommendations are outside of the scope of this paper and can be found elsewhere (Lee and Brusilovsky, 2012; Lee and Brusilovsky, 2014; Lee et al., 2011; Wongchokprasitti, 2015).

Note that while all kinds of recommendations look similar to the end user (each is provided with a ranked list of items), they are considerably different by their social nature. Content-based recommendations use only data from a target user, while tag-based and link-based recommendations extensively use data contributed by other users, which provides another example of social information access. It also means that just like all reviewed types of social information access, the latter kinds of recommendations rely considerably on the volume of user-contributed information. One of the goals of our study presented below was to determine whether we succeeded in reaching high enough levels of user engagement to generate sufficient volumes of "social wisdom."

\section{THE STUDY}

As described above, the CN3 system was designed to allow users to take advantage of various social features, as well as to support personal conference management (namely, scheduling). In turn, user contributions are extensively used to provide extended hyperspace and to create additional opportunities to find relevant information. To assess the value of these ideas and specific features, it is important to find out whether and to what extent these features are actually used.

This concept is directly connected to the question of whether the amount of user contributions is sufficient to support the social-navigation functionality of the system. In order to answer these questions, we collected and analyzed the transaction logs of CN3 while it is used in real conferences. In addition, in order to understand how users perceive and value various features, both in the system and the system as a whole, we conducted anonymous online surveys. Both approaches are recognized as a way to analyze and access the performance of conference support systems (Chin et al., 2012; Churchill et al., 2004; Cox et al., 2003; McDonald et al., 2008; Sumi and Mase, 2002). In this paper, we examine iConference2011, the largest conference among those where the CN3 system was deployed, and report the results of log analysis and user surveys collected at this conference.

\subsection{User Activity and Contributions}

At iConference 2011, the CN3 system was adopted as the main conference scheduling and social linking tool. In this capacity, $\mathrm{CN} 3$ accounts were automatically created for the conference registrants. However, in accordance with the conference privacy policies, those registrants who had not agreed to have their names to be published in the attendee list were not included for the automatic account creation. In total, 474 attendees opted to share their information at the time of registration. For each of these attendees, CN3 created a user account. One week prior to the conference, these users were introduced to $\mathrm{CN} 3$ by e-mail, along with instructions on how to access the system and reset the user's initial password. Out of these 474 attendees, 153 (32.3\%) logged on the system at least once and 109 bookmarked at least one conference presentation, which makes up 23\% of all attendees and $71.2 \%$ of those who logged on the system. 
Besides the attendees, other $\mathrm{CN} 3$ users also had access to the iConference (iConf) presentations (but did not have access to the list of attendees). CN3 is an open system, and therefore, while the conference attendees form the primary user group, other users can access 'public' information about the conference, including the program. In addition, any user who has an account in $\mathrm{CN} 3$ can bookmark presentations at any conference within the system. In total, at iConference 2011, there were 25 users outside the attendee group (hereafter called non-attendees) who bookmarked one or more iConference presentations. In total, 1574 bookmarks related to iConference presentations were recorded in the CN3 system. The 109 attendees created 1327 bookmarks and 25 nonattendees created 247 bookmarks. These figures are shown in Table 2.

Table 2. iConference bookmarking data

\begin{tabular}{|l|l|l|l|}
\hline & Total & Attendees & Non-Attendees \\
\hline $\begin{array}{l}\text { Users who created at least } \\
\text { one bookmark }\end{array}$ & 134 & 109 & 25 \\
\hline Total number of bookmarks & 1574 & 1327 & 247 \\
\hline $\begin{array}{l}\text { Average number of } \\
\text { bookmarks per user }\end{array}$ & 11.75 & 12.17 & 9.88 \\
\hline
\end{tabular}

Table 3. User activities in CN3

\begin{tabular}{|c|c|c|c|c|c|}
\hline \multirow[b]{2}{*}{ Activity type } & \multirow{2}{*}{$\begin{array}{l}\text { Number } \\
\text { of users }\end{array}$} & \multicolumn{4}{|c|}{ Number of activities } \\
\hline & & Total & $\begin{array}{l}\text { Before } \\
\text { iConf }\end{array}$ & $\begin{array}{l}\text { During } \\
\text { iConf }\end{array}$ & $\begin{array}{l}\text { After } \\
\text { iConf }\end{array}$ \\
\hline Bookmarking & 134 & 1574 & 1169 & 373 & 32 \\
\hline Tagging & 8 & 71 & 34 & 28 & 9 \\
\hline Following & 37 & 185 & 166 & 9 & 10 \\
\hline Connection request & 28 & 198 & 129 & 14 & 55 \\
\hline
\end{tabular}

These data are very important to demonstrate the feasibility of user bookmarks as a source of information for social navigation. It was one of our key concerns about whether $\mathrm{CN} 3$ can engage a sufficient number of users as contributors and whether the volume of contributions would be considerable enough to support social recommendation functionality. Given that the number of active contributors in social systems is generally relatively low, a conference context with an already low number of potential contributors presents a considerable challenge to social navigation technologies. However, the data shows that the $\mathrm{CN} 3$ system was able to engage a relatively large percentage of system attendees (about 23\%) as contributors, and even attracted contributions outside of the bounded set of attendees. The average number of contributions per contributor was also sufficiently large to enable a range of social techniques.

A more detailed time-based analysis of the users' contributory behavior (bookmarking and tagging iConference presentations, as well as linking to attendees) is shown in Table 3. The table shows that the largest fraction of the contributions was provided before the conference, a smaller fraction was provided during the conference, and a very small 
number of activities happened after iConference 2011. Day-by-day analysis of the bookmarking contributions (Figure 9) further shows that user bookmarking activity grows steadily, and reaches its peak during the two days that directly precede the conference. The activity level remains high during the first day of the conference, but drops rapidly afterwards.

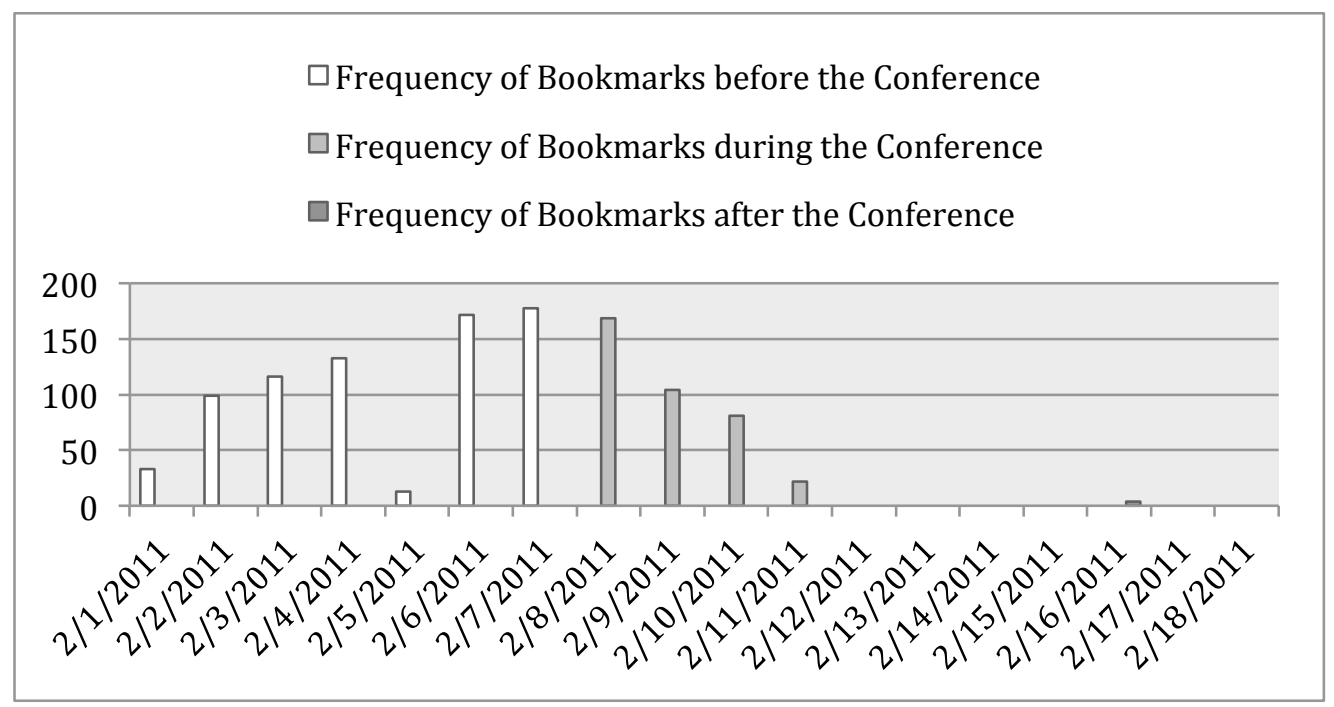

Figure 9. Number of bookmarks created per day before, during, and after the conference.

The observation that the majority of bookmarks were created before the conference is important for two reasons. First, it shows that rate of bookmark accumulation is quite high; as a result, social navigation and recommendation approaches based on bookmarks can start working relatively early, delivering a mature performance even before the start of the conference itself. Second, it shows that to be successful, a social conference system has to be released to the conference attendees well before the start of the event, in order to collect a sufficient volume of bookmarks that enable various social navigation pathways.

The usage statistics shown in Table 3 also reveals that out of the 153 attending users, $24 \%$ (37 users) followed one or more other users, and that $18 \%$ (28 users) tried to make a connection to other attendees. These users generated a good number of social linking attempts (185 following, 198 connection requests). This is a testament of a reasonably high interest in social linking features in CN3. However, among the 198 connection requests, about $79 \%$ remained unconfirmed. Multiple factors may contribute to this high number of unanswered requests. Some of these factors may be related to the nature of the system and its usage; there may have been a very short window available for the users to confirm a connection and an unequal participation rate. Other factors may relate to the visibility of connection requests in CN3. In the version used at iConference 2011, all requests were displayed on each user's personal profile page. Users did not see incoming connection requests unless they visited their own profile page, so some users might have not been aware of the requests. While placing the notifications of connection requests more prominently in the system and informing users by e-mail about new requests might 
increase the percentage of confirmed connections, this observation hints that unidirectional "following" connections might be preferable in short-term conference support systems, like CN3.

To place the participation number in a broader context, we compared the level of system usage and user activities observed in iConference 2011 to those observed at ASIS\&T 2010 (the pilot study setting) in order to evaluate whether the new design with enhanced social features encouraged more attendees to contribute. These two conferences are comparable in size and are attended by largely overlapping communities of information scientists. In this context, differences in participation levels could be attributed to the differences between the conference support systems. As shown in Table 4, we observed a substantial increase in both the number of users who bookmarked presentations and the average number of bookmarks per user. As a result, the total number of bookmarks increased almost three-fold after the switch from $\mathrm{CN} 2$ to $\mathrm{CN} 3$.

Table 4. Comparison of user activities in ASIS\&T 2010 (pre-study) and iConference 2011.

\begin{tabular}{|l|l|l|l|r|r|}
\hline & $\begin{array}{l}\text { Number } \\
\text { of } \\
\text { attendees }\end{array}$ & $\begin{array}{l}\text { Number of } \\
\text { users } \\
\text { bookmarked }\end{array}$ & $\begin{array}{l}\text { Number of } \\
\text { users } \\
\text { tagged }\end{array}$ & $\begin{array}{l}\text { Number of } \\
\text { bookmarks }\end{array}$ & $\begin{array}{l}\text { Average } \\
\text { number of } \\
\text { bookmarks } \\
\text { per user }\end{array}$ \\
\hline ASIST 2010 & 550 & $58(10.6 \%)$ & $11(2 \%)$ & 471 & 7.2 \\
\hline iConference 2011 & 474 & $109(23 \%)$ & $2(0.42 \%)$ & 1327 & 12.2 \\
\hline
\end{tabular}

\subsection{Log analysis}

In this section, we report the analysis of the logs collected from February 1 to February 18 , a period that begins a week before the conference started and ends a week after the conference concluded (as shown in Figure 9).

Our log program captures all the actions carried out by a user. Each time a new page is loaded as a user navigates through the system (for example, when the user goes to the list of attendees), it is logged with a timestamp, as well as with other parameters that represent relevant details (for example, the sorting criteria used to list the attendees). The system also logged a number of other actions (such as expanding/collapsing cells on the calendar view of the conference program and scheduling a presentation, among others). We use the term 'move' to refer to all possible user actions and navigation.

The moves roughly correspond to the main system features, since loading a page that provides a feature is recoded as a 'move' in the log. For example, one of the social features of $\mathrm{CN} 3$ enables users to see other users' scheduled presentations and their networks, and thereby provides opportunities to find useful contents and/or contacts. By examining the occurrences of "visiting someone else's profile page" move in the log, we can assess how often this social feature is actually used.

In the following analysis, we look at the distribution of user sessions and the frequency of individual user moves within those sessions. The dataset for the analysis includes 15,598 $\log$ records created between February 1, 2011 and February 18, 2011. Some abnormal 
records in the original log data (such as crawler visits) were removed in this dataset. Each record in the log represents a single user move. A user session is comprised of one or more moves, arranged in sequence. Our log system relies on PHP session support to distinguish user sessions. There are a total of 1,935 user sessions in the dataset, and the average number of moves per session is 8.06 .

Table 5 shows basic descriptive statistics about user moves, grouped by session categories. Login sessions refer to those in which the user went through the login process at some point during the session. Out of the total 1,935 user sessions, about $24 \%$ of these (468 sessions) were login sessions.

Table 5. Number of user moves per session, by session category.

\begin{tabular}{|l|l|l|l|l|l|l|}
\hline \multirow{2}{*}{ Session Category } & \multirow{2}{*}{$\mathrm{N}$} & \multirow{2}{*}{ Mean } & \multicolumn{3}{|l|}{ Quartile } & \multirow{2}{*}{ Range } \\
\cline { 4 - 7 } & & & 1 st & 2nd & 3 rd & \\
\hline All sessions & 1935 & 8.06 & 1 & 1 & 6 & $1-219$ \\
\hline Login sessions & 468 & 25.59 & 7 & 14 & 33 & $1-219$ \\
\hline Sessions with scheduling & 147 & 52.67 & 21 & 43 & 68 & $9-219$ \\
\hline
\end{tabular}

It is encouraging to observe a group of users who, although small in size, were highly engaged in their use of $\mathrm{CN} 3$, as they made a substantial number of moves per session. As mentioned in the introduction, any participatory system generally has a disproportionately large volume of contributions made by a small number of highly active users. While this rule is generally true for $\mathrm{CN} 3$, its fraction of actively engaged users is still much higher than might be expected, following the $1 \%$ rule cited above (van Mierlo, 2014). The large portion of anonymous sessions among the user sessions, however, poses a question. Some of those anonymous sessions involve a large number of moves, meaning that the users cared to spend time to use the system-yet did not log in to the system. As in any social system, the potential benefits of the social features in $\mathrm{CN} 3$ largely depend on open user participation and on the accumulation of user-generated information. The log data highlights the need for finding ways to better engage users of the $\mathrm{CN} 3$ system.

With the understanding of the overall composition of user sessions from iConference2011, we now examine the usage of individual features, captured as user moves. Our log program records detailed information at a fine-grained level. Given the limited number of login sessions where the full range of features can be observed, we decided to use a set of higher-level codes in order to see general patterns. For instance, the 'filtering' functionality of program visualization allows users to specify various conditions and highlight presentation(s) in the program that meet the condition. While the $\log$ records contain detailed information on the filtering condition, for the purposes of this analysis, we are concerned only with the category of the action-filtering. In the end, a set of 31 codes representing possible user moves in $\mathrm{CN} 3$ was created, and each log record was mapped to a code. The log table then was transformed into a text file, with each line containing the code for the corresponding user move, in sequence. 
In order to see the overall usage of features in $\mathrm{CN} 3$, we counted the frequency of the occurrences of each user move in the log file. Table 6 shows the frequency of key user moves, in descending order.

Note again that the main purpose of examining the frequency of user moves here is to see to what extent the features of interest are actually used. More specifically, we are interested in seeing to what extent users take advantage of the novel social features in CN3. Examples of these features include the accessibility to other users' schedules and contacts; the list of top entities; and the presentation detail page that shows the list of users who have scheduled the given presentation. Table 6 shows that both the features supporting personal use (for example, program viewing, expanding program cells to check individual presentations) and the features providing social awareness regarding other users and their activities (for example, top entities, user pages) are frequently used.

Table 6. Frequency of all user moves

\begin{tabular}{|c|c|}
\hline Move & Frequency \\
\hline Conference program & 3158 \\
\hline Presentation detail & 2044 \\
\hline Expand a program cell & 1941 \\
\hline User profile page & 1184 \\
\hline Scheduling a presentation & 1124 \\
\hline Top entities & 833 \\
\hline Collapse a program cell & 474 \\
\hline Search & 385 \\
\hline View by click (in program) & 209 \\
\hline Unscheduling a presentation & 111 \\
\hline
\end{tabular}

The social features in $\mathrm{CN} 3$ also provide new linking and navigation paths for users. As described in Section 3, the entity names shown in the 'top', 'users', and 'presentation details' pages are clickable links, meaning that, for instance, while checking another user's page a user may click one of the presentations scheduled by the person or one of the social connections of the person. In order to see the extent that users take advantage of these types of 'socially enabled' navigation paths, we looked at the sequence of user moves in the log. For example, let us examine the 'Top' page, which provides the list of the most frequently scheduled presentations and the most active users. As shown in Table 6 , there were a total of 833 cases of 'Top' page visits. In 102 cases, the 'Top' page was the final sequence of the given session, with no next moves. Out of the remaining 781 cases, 144 were followed by the 'User' page and 120 by the 'Presentation detail' page. That is, in about $34 \%$ of cases, users follow social navigation paths. In the remaining $66 \%$ of cases, users move back to the program, the attendee list, their own schedule (myCN3), or to one of the other pages. 
A more notable pattern was found in the sequence of user moves that followed a visit to the 'User' pages. Among the 1,184 cases of 'User' page visits, only 31 cases were the final move of the given session, leaving 1,153 cases with additional moves. In 492 cases $(42.7 \%)$, the next move after a user page was to another user page, meaning that the users checked the social connections of other users. It shows that social links contributed by users and leveraged by $\mathrm{CN} 3$ as navigation pathways emerged as an important tool for user exploration. In 71 cases $(6.2 \%)$ a visit to a 'User' was followed by a 'Presentation detail' page, while 33 cases $(2.8 \%)$ was directly followed by 'Scheduling.' This shows that in about $9 \%$ of cases, users found an interesting presentation when they visited another user's page, which led them to either check the details of the presentation or even directly add it to their own schedule. Both patterns show a relatively high usage of the key social navigation features that are provided by $\mathrm{CN} 3$.

\subsection{Survey Results}

The online survey was distributed on February 28, 2011 to 105 iConference attendees who bookmarked at least one presentation in CN3. The survey ran until March 18, 2011, resulting in 35 responses (33.3\% response rate).

The survey asked three broad categories of questions. First, questions were asked about the perceived usefulness of the system as a whole, as well as individual features. Second, questions were asked about the awareness of the features and the degree to which features were visible or easy to find. Third, questions were asked about the overall relevance of recommendations provided by the system.

Out of $35 \mathrm{CN} 3$ users who answered our online survey, $60 \%$ replied that $\mathrm{CN} 3$ made easier to navigate the conference program, and $40 \%$ replied that the application helped them to participate more effectively in the conference.

Table 7. Awareness about CN3 features

\begin{tabular}{|l|l|l|}
\hline Awareness that you can... & Yes (\%) & No (\%) \\
\hline edit profile and privacy settings & 66 & 34 \\
\hline see top items & 63 & 37 \\
\hline see others' schedule & 83 & 17 \\
\hline see who else scheduled a presentation & 80 & 20 \\
\hline contact/follow other users & 87 & 13 \\
\hline compare your contacts' schedule and yours & 20 & 80 \\
\hline receive presentation recommendations & 53 & 47 \\
\hline receive people recommendations & 33 & 67 \\
\hline
\end{tabular}

Overall awareness of the socially-enabled CN3 features varies from feature to feature. Seeing others' schedules and who else have scheduled certain presentations were among the most noticed features, $83 \%$ and $80 \%$ respectively. Most people knew about the ability to connect with or follow other users $(87 \%)$, and $63 \%$ knew that the most popular items 
were accessible in the system. Two-thirds $(66 \%)$ of users were aware that they could edit their profile and privacy settings, and $53 \%$ knew that they could receive presentation recommendations (see Table 7). However, less prominent and novel features, such as people recommendations and the ability to compare schedules, were much less known; only $33 \%$ and $20 \%$ of users were aware of their existence, respectively.

We interpret these results as evidence that the majority of respondents noticed features that had clear affordances and were the ones that the users were more likely to expect in a social information system. This finding stresses the need to provide better affordance and even direct recommendations of innovative features that users might not expect to see in this new kind of system.

The majority of the respondents considered most of the key CN3 features to be useful (Table 8). It was very encouraging to see the list of most useful features topped by two social navigation features provided by the system - seeing others' schedules and who else has bookmarked a presentation (74\% and $70 \%$, respectively). The ability to see 'top' items was also assessed positively (54\% of users). To account for the fraction of users who disagreed that a specific feature is useful, we calculated a positive balance (the percentage of agreeing users minus the percentage of disagreeing users) for each feature.

The balance analysis shows that the usefulness of the key social features was much higher than the usefulness of classic features, like list program view. While the data also shows that the users were less enthusiastic about some novel features, such as comparing the user's schedule to other users' schedules (only 36\% of users considered it useful and $53 \%$ of them were not sure), this result appears to be an outcome of a feature that remained unnoticed by the majority of users, as shown in Table 7. Among users who knew about this feature, $50 \%$ found it useful, and 50\% had a neutral opinion, and none was negative, resulting in a positive balance of 50 as well.

Table 8. User feedback: usefulness of CN3 features.

\begin{tabular}{|l|l|l|l|l|}
\hline Usefulness of... & $\begin{array}{l}\text { Agree } \\
(\mathbf{\%})\end{array}$ & $\begin{array}{l}\text { Neither } \\
(\mathbf{\%})\end{array}$ & $\begin{array}{l}\text { Disagree } \\
(\%)\end{array}$ & $\begin{array}{l}\text { Positive } \\
\text { balance }\end{array}$ \\
\hline Calendar visualization & 60 & 31 & 9 & 51 \\
\hline List view & 50 & 28 & 22 & 28 \\
\hline Top items view & 54 & 36 & 10 & 44 \\
\hline Seeing others' schedule & 74 & 19 & 7 & 67 \\
\hline $\begin{array}{l}\text { Seeing who else scheduled a } \\
\text { presentation }\end{array}$ & 70 & 20 & 10 & 60 \\
\hline $\begin{array}{l}\text { Compare your contacts' schedule } \\
\text { and yours }\end{array}$ & 36 & 53 & 10 & 26 \\
\hline
\end{tabular}

When rating the features specifically by their support in finding interesting presentations, users singled out the calendar visualization as the most useful feature (78\%). The rating of other social features varied from $25 \%$ to $34 \%$, which indicates that these features play an important secondary role in information access. Visiting others' schedule and seeing 
the top items were selected as useful by $34 \%$ and $31 \%$ of users. Filtering by contacts and followed users schedules were mentioned by $25 \%$ and $19 \%$ of respondents, respectively. Filtering by name and using the search box were considered to be useful by $25 \%$ and $19 \%$, respectively. See Table 9 for the summary of these results. It is interesting to observe that the overall usefulness rating of the same social features is much higher in all cases. This hints that in user perception, the information access value of the social features is only a part their total value, and that they might be important to users for a number of other reasons such as social orientation and awareness, among others.

Table 9. User feedback: usefulness of features in finding presentations.

\begin{tabular}{|l|l|}
\hline Usefulness in finding presentations & $\mathbf{\%}$ \\
\hline Browsing program using calendar visualization & 78 \\
\hline Browsing program using list view & 41 \\
\hline Visiting another person's schedule & 34 \\
\hline Visiting the list of top items & 31 \\
\hline Filtering by schedule of people I'm following & 25 \\
\hline Filtering by my contact's schedule & 19 \\
\hline Filtering by author's name & 25 \\
\hline Using search box & 19 \\
\hline
\end{tabular}

Table 9. Recommendation feedback.

\begin{tabular}{|l|l|l|l|}
\hline Relevance of... & Frequently (\%) & Occasionally (\%) & Rarely (\%) \\
\hline $\begin{array}{l}\text { presentation } \\
\text { recommendations }\end{array}$ & 12.5 & 50 & 37.5 \\
\hline people recommendations & 30 & 30 & 40 \\
\hline
\end{tabular}

Finally, the analysis of user questions related to recommendation functionality brought mixed results. On one hand, the majority of respondents indicated that they are interested in receiving both presentation and people recommendations ( $80 \%$ and $73 \%$, respectively), while few users stated that they wouldn't be willing to receive those types of recommendations (7\% and 3\%, respectively). Moreover, to get these benefits, $50 \%$ of users would be willing to provide information from external social networks (against $17 \%$ of users who would not be willing to provide such information). At the same time, user awareness about the current presentation and people recommendation was low (see Table 7) and the quality of the recommendations was far from perfect. Only $12.5 \%$ of users who were aware of the existence of presentation recommendations found presentation recommendations to be frequently relevant, and $37.5 \%$ found them to be rarely relevant. Among users who knew about the people recommendation feature, 30\% considered them to be frequently relevant, and $40 \%$ found them to be rarely relevant (see Table 9). This data confirms that recommendation is a highly valuable feature in a 
conference support system, but also stresses the need to provide higher visibility for this functionality and invest in increasing the quality of recommendations.

\section{DISCUSSION AND FOLLOW-UP WORK}

The goal of our project was to develop an integrated social system that can support researchers attending academic conferences. In addition to core functionalities like the conference program and list of attendees, the system piloted a number of innovative social features that were designed to enhance the users' conference experience and help conference attendees to find interesting presentations and colleagues. Our study, performed in the context of iConference 2011, attempted to answer some key research questions associated with the Conference Navigator system and the use of social navigation and linking at academic conferences. Our main goal was to investigate to what extent such a system would be both appealing and useful for the conference attendees, and whether the volume of social contributions generated by such a system's users would be sufficient to fuel its social mechanisms. We were also interested to find out to what extent each of the system's innovative features would be used and positively rated by the users.

The results of a $\mathrm{CN} 3$ deployment at iConference demonstrated a solid level of user interest and a larger level of active contribution than we expected. It is commonly known that only a small fraction of the potential users of typical social information systems (for example, a movie recommender or a research paper tagging system) contribute to the systems. Moreover, even a smaller fraction of these will emerge as active users. Research papers frequently cite a $1 \%$ rule of active users, which indicates that $90 \%$ of users only observe or read content, $9 \%$ contribute only sparsely, and $1 \%$ create the vast majority of the content (van Mierlo, 2014). The participation rate for CN3 was much higher, as 153 out of 474 users logged on the system at least once. While even this could be considered to be a high participation ratio in social information systems, the overall number of users was even higher, given that a large number of actions in the system were generated by people who used the system without logging in (see Table 5). Moreover, among registered users, a relatively large fraction emerged as contributors: 109 out of 153 logged-in users bookmarked at least one conference presentation. Comparing this data with data obtained in a pilot study performed with an earlier version of the system in a similar context provided evidence that the innovative features introduced in CN3 engaged its users to a larger degree and engaged more users overall (see Table 4).

We believe that these results demonstrate the value and feasibility of maintaining social information systems for academic conferences. We can hypothesize that the immediate value of the system is in generating a relatively large (for social information systems) level of usage and contribution that compensates for the relatively small number of potential users and a small period of time for them to contribute. At the same time, we clearly see the effect of time constraints on the utility of the CN3 system. Less than a quarter of all contributions were made during the few days of the conference, while about three-quarters of contributions were made before the start of the conference. The data shows that it is critical to introduce the system to potential users at least one week before the conference, preferably well in advance. A natural time to introduce such a system is when the conference program is made public by the conference chairs. We hypothesize 
that an earlier release of the system will increase user chances to explore both the program and the prospective attendees, and will produce a higher level of contributions.

While the overall usage of the system was relatively high, the data shows that use of the system was not distributed equally among users. As in other social systems, there were many users who did very little and a few who did a lot. While it was encouraging to see a considerable number of users using the system heavily and generating many contributions, there were still many users who just touched the system, which is also indicated by the relatively large number of short sessions. It is a challenge for the future versions of the system to more fully engage these users.

A related problem is the large number of users who worked within the system without logging in. As the log analysis shows, users who were not logged in generated the majority of user sessions. This creates a double problem for the system. On one hand, users who are not logged in cannot generate social contributions, such as bookmarks and user connections). On the other hand, many important features such as scheduling and recommendation were only available to users who logged in. Users who do not log in cannot see these features, which likely decreased their motivation to work with the system (as shown by a much smaller average number of moves for non-logged-in users). The logging-in bottleneck is a known problem in the area of social and personalized systems and must be addressed.

The log analysis also demonstrated that the balance of user presentation-centered and people-centered navigation is not equal. The main volume of user information access was focused on program and presentation information. While the list of attendees and user pages were also popular, the number of actions related to user information access totaled to less than $50 \%$ of the number of actions related to presentation information access. On the level of contributions, the difference was even higher: the number of created or requested social links was about four times smaller than the number of bookmarks.

Clearly, the most useful value of the Conference Navigator system at iConference 2011 was access to information about the conference program and presentations. It could be argued that presentations are more important than social contacts at the conference. More likely, however, the majority of social networking happened outside of CN3 and was simply not captured by the system. This stresses the importance of integrating tag-based approaches for tracking user social activity, as explored in (Atzmüller et al., 2011; Cattuto et al., 2010; Chin et al., 2012).

While the social linking functionality was underused, the presence of users as first-class objects in the system was very important. The analysis of user feedback demonstrates that the users appreciated the several innovative social features based on user contributions. The ability to explore the schedules of other users and to see who scheduled a specific presentation (namely, two-way connections between presentations and attendees, as established by processing community contributions) were rated as the two most useful features of the system. The top items view was also found to be useful by more than half of respondents. Log data supports this finding, showing the relatively high usage of these features. At the same time, some most sophisticated social features, such as the ability to compare two schedules or to filter the schedule using one's contacts were less appreciated and less used than simpler and more transparent features that supported the 
same need (such as, one can compare one's own schedule with a schedule of another user by simply exploring the other user's schedule, as provided on the user profile page).

While this data might suggest that there is little reason to offer sophisticated features, we believe that a low level of appreciation of these features was mostly the results of the users' low awareness of them. Features like schedule comparison or presentation recommendations were rather unusual and not very easy to notice. The users who did not expect these features to be offered could simply miss seeing them or not understand their presence and as a result, did not consider them to be useful.

As the awareness feedback shows, only $20 \%$ of users were aware of the schedule comparison feature and only 53\% of users were aware of the presentation recommendation feature. The feature complexity and less prominent position in the interface contributed to this effect. Finally, an additional factor that decreased the appreciation of advance features was the need to login to see these features. As previously mentioned, the majority of user sessions were created without logging in, which allowed the users to see the core functionality, but not the advanced features.

In follow-up work with CN3 that has been performed since 2012 and is currently being performed, we attempted to address a number of problems discovered by our iConference study and examine additional approaches to social linking and social navigation. Some of this work has been reported, while more work is still ongoing. As previously mentioned, we explored the prospects of offering social navigation by using both direct user contributions and user activity on the conference floor, as tracked by badges. In collaboration with the Conferator team (Atzmüller et al., 2011), we created a new version of the Conferator system where presentation exploration and scheduling were supported by the new version of TalkRadar based on CN3, while social linking were supported by the original badge-enabled PeerRadar system. This collaboration brought some interesting results that were presented in (Macek et al., 2012; Scholz et al., 2014).

We are also exploring alternative social visualization approaches to help users in finding relevant presentations. TalkExplorer view used set-based visualization to support exploration of conference presentation in relationships to users who scheduled them, their tags, and direct recommendations (Verbert et al., 2013). SetFusion view used a similar approach to increase the visibility and usage of presentation recommendations (Parra and Brusilovsky, 2015). A good portion of follow-up work also focused on developing better approaches for recommending conference presentations (Lee and Brusilovsky, 2014; Wongchokprasitti, 2015). CN3 remains an ongoing project, and we hope that our work with the system will lead to better understanding how efficient conference support systems should be designed and operated, as well as provide deeper insights about user information and social behavior during a conference (Macek et al., 2012).

\section{ACKNOWLEDGEMENTS}

This work has been partially supported by an NSF award IIS 1052768 to the first two authors. The third and the fourth authors have been supported by Chilean Scholarship from the National Commission for Science Research and Technology (CONICYT, Chile). 


\section{REFERENCES}

M. Atzmüller, D. Benz, S. Doerfel, A. Hotho, R. Jäschke, B.E. Macek, F. Mitzlaff, C. Scholz and G. Stumme, 2011. Enhancing Social Interactions at Conferences. Information Technology, 53 (3), 101-107.

A. Bhardwaj, J. Kim, S. Dow, D. Karger, S. Madden, R. Miller and H. Zhang, 2014. Attendee-Sourcing: Exploring The Design Space of Community-Informed Conference Scheduling. Second AAAI Conference on Human Computation and Crowdsourcing.

R. Borovoy, F. Martin, S. Vemuri, M. Resnick, B. Silverman and C. Hancock, 1998. Meme Tags and Community Mirrors: Moving from Conferences to Collaboration. Proceedings of the 1998 ACM Conference on Computer Supported Cooperative Work ACM, Seattle, Washington, USA, 159-168.

P. Brusilovsky, 2008. Social Information Access: The Other Side of the Social Web. In: V. Geffert, et al., eds., SOFSEM 2008, 34th International Conference on Current Trends in Theory and Practice of Computer Science Lecture Notes in Computer Science 4910, Springer Verlag, High Tatras, Slovakia, 5-22.

P. Brusilovsky, D. Parra, S. Sahebi and C. Wongchokprasitti, 2010. Collaborative Information Finding in Smaller Communities: The Case of Research Talks. 6th International ICST Conference on Collaborative Computing: Networking, Applications and Worksharing, Chicago, Illinois, USA

J. Carroll and M.B. Rosson, 2013. Wild at home: the neighborhood as a living laboratory for HCI. ACM Transactions on Computer-Human Interaction 20 (3), 16.

C. Cattuto, W. Van den Broeck, A. Barrat, V. Colizza, J.-F. Pinton and A. Vespignani, 2010. Dynamics of person-to-person interactions from distributed RFID sensor networks. Dynamics of Person-to-Person Interactions from Distributed RFID Sensor Networks, 5 (7), e11596.

A. Chin, B. Xu, F. Yin, X. Wang, W. Wang, X. Fan, D. Hong and Y. Wang, 2012. Using Proximity and Homophily to Connect Conference Attendees in a Mobile Social Network. 32nd International Conference on Distributed Computing Systems Workshops (ICDCSW 2012) IEEE, 79-87.

E. Churchill, A. Girgensohn, L. Nelson and A. Lee, 2004. Blending digital and physical spaces for ubiquitous community participation. Communications of the ACM, 47 (2), 38-44.

D. Cox, V. Kindratenko and D. Pointer, 2003. IntelliBadge ${ }^{\mathrm{TM}}$ : Towards Providing Location-Aware Value-Added Services at Academic Conferences UbiComp 2003: Ubiquitous Computing. In: A. Dey, A. Schmidt and J. McCarthy, eds. IntelliBadge TM: Towards Providing Location-Aware Value-Added Services at Academic Conferences UbiComp 2003: Ubiquitous Computing. 2864. Springer Berlin / Heidelberg,264-280.

J.N. Cummings, B.S. Butler and R. Kraut, 2002. The quality of online social relationships. Commun. ACM, 45, 103-108.

A. Dey, D. Salber, G. Abowd and M. Futakawa, 1999. The Conference Assistant: combining context-awareness with wearable computing. Wearable Computers, 1999. Digest of Papers. The Third International Symposium on IEEE, 21-28. 
A. Dieberger, P. Dourish, K. Höök, P. Resnick and A. Wexelblat, 2000. Social navigation: Techniques for building more usable systems. interactions, 7 (6), 3645.

N.B. Ellison, 2007. Social network sites: Definition, history, and scholarship. Journal of Computer-Mediated Communication, 13 (1), 210-230.

S. Farnham, P. Brown and J. Schwartz, 2009. Leveraging social software for social networking and community development at events. Proceedings of the fourth international conference on Communities and technologies ACM, University Park, PA, USA, 235-244.

S. Farrell, C. Campbell and S. Myagmar, 2005. Relescope: an experiment in accelerating relationships. CHI '05 extended abstracts on Human factors in computing systems ACM, Portland, OR, USA, 1363-1366.

R. Farzan and P. Brusilovsky, 2007. Community-based Conference Navigator. In: V. Dimitrova, M. Tzagarakis and J. Vassileva, eds., 1st Workshop on Adaptation and Personalisation in Social Systems: Groups, Teams, Communities at the 11th International Conference on User Modeling, UM 2007, Corfu, Greece, 30-39.

R. Farzan and P. Brusilovsky, 2008. Where did the Researchers Go? Supporting Social Navigation at a Large Academic Conference. The 19th ACM Conference on Hypertext \& Hypermedia, Pittsburgh, Pennsylvania, USA, 203-211.

J. Kim, H. Zhang, P. André, L. Chilton, W. Mackay, M. Lafon, R. Miller and S. Dow, 2013. Cobi: A Community-informed Conference Scheduling Tool. Proceedings of the 26th Annual ACM Symposium on User Interface Software and Technology ACM, St. Andrews, Scotland, United Kingdom, 173-182.

S.i. Konomi, S. Inoue, T. Kobayashi, M. Tsuchida and M. Kitsuregawa, 2006. Supporting Colocated Interactions Using RFID and Social Network Displays. IEEE Pervasive Computing, 5, 48-56.

P. Kraker, P. Weißensteiner and P. Brusilovsky, 2014. Altmetrics-based Visualizations Depicting the Evolution of a Knowledge Domain. 19th International Conference on Science and Technology Indicators (STI 2014), Leiden, NL.

R. Kraut and P. Resnick, 2012. Building Successful Online Communities: EvidenceBased Social Design, Cambridge, MA: MIT Press.

D. Lee and P. Brusilovsky, 2012. Exploring Social Approach to Recommend Talks at Research Conferences. COLLABORATECOM 2012 - 8th IEEE International Conference on Collaborative Computing: Networking, Applications and Worksharing, Pittsburgh, USA.

D. Lee and P. Brusilovsky, 2014. Recommending Talks at Research Conferences Using Users' Social Networks. International Journal of Cooperative Information Systems, 23 (2).

D. Lee, P. Brusilovsky and T. Schleyer, 2011. Recommending Future Collaborators using Social Features and MeSH terms. the 74th Annual Meeting of the American Society for Information Science and Technology, New Orleans, Louisiana, USA.

B. Macek, C. Scholz, M. Atzmueller and G. Stumme, 2012. Anatomy of a Conference. Proceedings of the 23rd ACM Conference on Hypertext and Social Media ACM, Milwaukee, Wisconsin, USA, 245-254.

J. McCarthy, D. McDonald, S. Soroczak, D. Nguyen and A. Rashid, 2004. Augmenting the social space of an academic conference. Proceedings of the 2004 ACM 
conference on Computer supported cooperative work ACM, Chicago, Illinois, USA, 39-48.

D. McDonald, J. McCarthy, S. Soroczak, D. Nguyen and A.M. Rashid, 2008. Proactive displays: Supporting awareness in fluid social environments. ACM Transactions on Computer-Human Interaction 14 (4), 1-31.

S.M. McNee, N. Kapoor and J. Konstan, 2006. Don't look stupid: avoiding pitfalls when recommending research papers. the 20th Anniversary Conference on Computer Supported Cooperative Work Banff, Alberta, Canada, 171-180.

S.E. Middleton, N.R. Shadbolt and D.C. De Roure, 2004. Ontological User Profiling in Recommender Systems. ACM Transactions on Information Systems, 22 (1), $54-$ 88.

D. Parra and P. Brusilovsky, 2015. User-controllable personalization: A case study with SetFusion. International Journal of Human-Computer Studies, 78, 43-67.

M.J. Pazzani and D. Billsus, 2007. Content-based recommendation systems. In: P. Brusilovsky, A. Kobsa and W. Neidl, eds. Content-based recommendation systems. Lecture Notes in Computer Science 4321. Berlin Heidelberg New York: Springer-Verlag,325-341.

M. Pham, D. Kovachev, Y. Cao, G.M. Mbogos and R. Klamma, 2012. Enhancing Academic Event Participation with Context-aware and Social Recommendations. Advances in Social Networks Analysis and Mining (ASONAM), 2012 IEEE/ACM International Conference on IEEE, 464-471.

G. Salomon, 1990. Designing Casual-user Hypertext: The CHI'89 InfoBooth. Proceedings of the SIGCHI Conference on Human Factors in Computing Systems ACM, Seattle, Washington, USA, 451-458.

C. Scholz, J. Illig, M. Atzmueller and G. Stumme, 2014. On the Predictability of Talk Attendance at Academic Conferences. Proceedings of the 25th ACM Conference on Hypertext and Social Media ACM, Santiago, Chile, 279-284.

K. Sugiyama and M. Kan, 2010. Scholarly paper recommendation via user's recent research interests. Proceedings of the 10th annual joint conference on Digital libraries ACM, Gold Coast, Queensland, Australia, 29-38.

Y. Sumi and K. Mase, 2001. Digital Assistant for Supporting Conference Participants: An Attempt to Combine Mobile, Ubiquitous and Web Computing. Ubicomp 2001: Ubiquitous Computing, Third International Conference Springer, Atlanta, Georgia, USA, 156-175.

Y. Sumi and K. Mase, 2002. Conference assistant system for supporting knowledge sharing in academic communities. Interacting with Computers, 14 (6), 713-737.

T. van Mierlo, 2014. The $1 \%$ rule in four digital health social networks: an observational study. , 16(2). Journal of medical Internet research, 16 (2).

K. Verbert, D. Parra-Santander, P. Brusilovsky and E. Duval, 2013. Visualizing Recommendations to Support Exploration, Transparency and Controllability. the 2013 International Conference on Intelligent User Interfaces, IUI '2013 ACM Press, Santa Monica, USA, 351-362.

J. Wang, D. Soroker and C. Narayanaswami, 2010. Event maps: a collaborative calendaring system for navigating large-scale events. Proceedings of the 28th of the international conference extended abstracts on Human factors in computing systems ACM, Atlanta, Georgia, USA, 3691-3696. 
F. Windhager, M. Smuc and L. Zenk, 2014. Conferences, Coffee and Complexity: Supporting Cognition and Communication at Conventions. 2014 18th International Conference on Information Visualisation (IV) IEEE, 78-83.

C. Wongchokprasitti, Using External Sources to Improve Research Ralk Recommendation in Small Communities PhD Thesis. University of Pittsburgh, 2015.

C. Wongchokprasitti, P. Brusilovsky and D. Parra, 2010. Conference Navigator 2.0: Community-Based Recommendation for Academic Conferences. Workshop on Social Recommender Systems at the International Conference on Intelligent User Interfaces (IUI 2010), Hong Kong, China.

N. Yaw Asabere, F. Xia, W. Wang, J. Rodrigues, F. Basso and J. Ma, 2014. Improving Smart Conference Participation Through Socially Aware Recommendation. Human-Machine Systems, IEEE Transactions on, 44 (5), 689-700. 